\title{
A categorical action on quantized quiver varieties
}

\author{
Ben Webstenl] \\ Department of Mathematics \\ Northeastern University \\ Boston, MA \\ Email: b.webster@neu.edu
}

\begin{abstract}
In this paper, we describe a categorical action of any symmetric Kac-Moody algebra on a category of quantized coherent sheaves on Nakajima quiver varieties. By "quantized coherent sheaves," we mean a category of sheaves of modules over a deformation quantization of the natural symplectic structure on quiver varieties. This action is a direct categorification of the geometric construction of universal enveloping algebras by Nakajima.
\end{abstract}

\section{Contents}

1. The 2-category $\mathcal{U}$

2. Quiver varieties

3. Hecke correspondences and categorical actions

4. The proof of Theorem 3.1

References

Let $\mathfrak{g}$ be an arbitrary Kac-Moody algebra with symmetric Cartan matrix, and $\Gamma$ its associated Dynkin graph. Nakajima showed that there exists a remarkable connection between the algebra $U(\mathfrak{g})$ and certain varieties, called quiver varieties, constructed directly from the graph $\Gamma$. This construction takes the form of a map from $U(\mathrm{~g})$ to the Borel-Moore homology of a quiver analogue of the Steinberg variety [Nak98].

Both the source and target of this map have natural categorifications:

- the algebra $\mathcal{U}(\mathfrak{g})$ is categorified by a 2-category $\mathcal{U}$. Actually several variations on the theme of this category have been introduced by Rouquier [Roua], Khovanov-Lauda [KL10], and Cautis-Lauda [CL15]; we will use the formalism of the last of these. A 2-functor from this category into another 2-category is called a categorical action of $\mathfrak{g}$ in this 2-category.

- the Borel-Moore homology of the "Steinberg" of a symplectic resolution $\mathfrak{M}$ (such as a quiver variety) is categorified by a certain category of sheaves on $\mathfrak{M} \times \mathfrak{M}$. The structure sheaf of $\mathfrak{M}$ possesses a quantization, in the sense of [BK04], and the category of interest to us is that of bimodules over this quantization which satisfy a "Harish-Chandra" property, as described by Braden, Proudfoot

\footnotetext{
${ }^{1}$ Supported by the NSF under Grant DMS-1151473 and by the NSA under Grant H98230-10-1-0199.
} 
and the author [BPW, §6.1-2]. Viewed correctly, these bimodules on the quiver varieties associated to a single highest weight $\lambda$ can be organized into a 2category, which we denote $Q^{\lambda}$.

Thus, this previous work suggests how to categorify Nakajima's map:

Theorem A For each highest weight $\lambda$, there is a categorical representation of $\mathfrak{g}$ in the 2 -category $Q^{\lambda}$; taking "characteristic cycles" of these bimodules recovers the geometric construction of Uं by Nakajima.

Furthermore, the form of this functor is strongly suggested by Nakajima's work; his map is defined by sending the Chevalley generators of $U(\mathfrak{g})$ to particular correspondences, called Hecke correspondences, which have natural moduli-theoretic significance. We "upgrade" these correspondences to modules over deformation quantizations, and show that these satisfy the categorical analogues of the Chevalley presentation. For the experts, we should note that this will not work with arbitrary quantizations. The quantizations we wish to consider are classified up to isomorphism by classes in $H^{2}(\mathfrak{M} ; \mathbb{C})$ called periods. The correspondences can only be quantized when the period satisfies an integrality condition, as we'll discuss in much more detail in Section 3.1 .

We regard this theorem as very strong evidence of the naturality of the notion of a categorical $\mathfrak{g}$-action currently circulating in the literature. While defined diagrammatically in a way that might outwardly seem arbitrary, in fact, its relations are hard-coded in the geometry of quiver varieties.

This action of a 2-category is also quite useful in understanding categories of sheaves on quiver varieties. In particular, we'll use it to understand the category of core modules for certain "integral" quantizations. These are closely related to the category of finite dimensional modules over a global quantization of the quiver variety. Work of Bezrukavnikov and Losev [BL] following up on this paper has described this category for more general quantizations, resolving a conjecture of Etingof on the structure of finite dimensional modules over a symplectic reflection algebra.

This theorem fits into a context of older results. Very close analogues of the functors that appear in this representation have already been constructed in work of Zheng [Zhe] and Li [Lia, Lib]. However, these authors work in a slightly different context, which is based on constructible sheaves rather than deformation quantizations. The Riemann-Hilbert correspondence has already established a tie between constructible sheaves on a space $X$, and certain modules over a deformation quantization of $T^{*} X$ : the differential operators $\mathcal{D}_{X}$ on $X$. From this perspective, if there were a space $Y$ of which a given Nakajima quiver variety were the cotangent bundle (there almost never is) then sheaves of modules over the quantized structure sheaf could be thought of as a replacement for the category of D-modules on the hypothetical space $Y$. As 
pointed out by Zheng [Zhe, §2.2], his work was in a sense intended to understand constructible sheaves with the same philosophy.

Rouquier [Roub, 5.10] showed that Zheng's action can be strengthened to an action of two 2-category $\mathcal{U}$; while it is not obvious that Rouquier's category is the same as that from [CL15], this was later proven by Brundan [Bru]. Rouquier's result is extremely close to the first clause of Theorem $\mathrm{A}$, but a host of annoying details rise up if one tries to derive one from the other: the result [Roub, 5.10] only establishes that the functors induce an action on a subcategory of Zheng's category, though this proof could likely be extended; all the above work is on $\mathbb{Q}_{\ell}$-sheaves on a variety over finite fields rather than over $\mathbb{C}$, etc. None of these issues are insuperable, but we felt the reader would be better served by an exposition which is more native to the world of deformation quantizations.

We are also motivated by analogous results that have appeared in the literature on coherent sheaves, for example in the work of Cautis, Licata and Kamnitzer [CK, CKL10, CKLa, CKLb, CKLc]. Amongst other things, these results show that the categories of coherent sheaves on quiver varieties carry a version of a categorical action. In particular, these results have lead to interesting equivalences between derived categories of coherent sheaves. From our perspective, the action on sheaves over deformation quantizations is easier to work with, since one can use topological methods for D-modules, and seems to be the more basic object. In forthcoming work, Cautis, Dodd and Kamnitzer [CDK] will make between classical and quantum situations precise, showing that the action on coherent sheaves of quiver varieties in [CKLb] is a classical limit of the action presented here.

More generally, this action is but one aspect of close ties between the geometry of quiver varieties and the theory of categorical Lie algebra actions. It builds on work of Rouquier, Varagnolo and Vasserot [Roub, VV11] and is expanded further in further work of the author [Webe], which relates other categories of modules over these deformation quantizations to known categorical g-actions.

Another perspective on these deformation quantizations is that they provide a replacement for the Fukaya category of a complex symplectic variety. Such a connection is suggested by Kapustin and Witten [KW07, \$11] from a physical perspective, and the work of Nadler and Zaslow [NZ09] relating constructible sheaves and the Fukaya category of a cotangent bundle is also quite suggestive along these lines. In particular, it would be very interesting to find a categorical Lie algebra action in the 2-category of Lagrangian correspondences constructed by Wehrheim and Woodward [WW10]. Hopefully, instead of finding modules supported on the Hecke correspondences, one would simply consider them as objects in the Fukaya category.

Our main technical tool is a theorem of Rouquier [Roub, 4.13] which greatly reduces the number of relations which need to be checked in order to confirm that a candidate is a categorical action. This result is quite similar to earlier works of Chuang 
and Rouquier ([CR08, 5.27] \& [Roua, 5.27]) and Cautis and Lauda [CL15, Th. 1.1], which likewise reduce the number of calculations needed, but which require stronger hypotheses. In particular, we can rely on calculations of Varagnolo and Vasserot from [VV11] for the most important check of relations between 2-morphisms; the other conditions either follow from general principles or are close analogues of results proven by Zheng and $\mathrm{Li}$, with proofs that can be adapted.

Acknowledgements. This paper owes a great debt to Yiqiang Li; his work was an important inspiration, and he very helpfully pointed out a serious mistake in a draft version. I also want to thank Nick Proudfoot, Tony Licata and Tom Braden; I depended very much on previous work and conversations with them to be able to write this paper. I thank Sabin Cautis and Aaron Lauda for sharing an early version of their paper with me. I also appreciate very stimulating conversations with Catharina Stroppel, Ivan Losev and Peter Tingley.

Notation. We let $\Gamma$ be an oriented graph and $\mathfrak{g}$ the associated Kac-Moody algebra. Consider the weight lattice $Y(\mathfrak{g})$ and root lattice $X(\mathfrak{g})$, and the simple roots $\alpha_{i}$ and coroots $\alpha_{i}^{\vee}$. Let $c_{i j}=\alpha_{j}^{\vee}\left(\alpha_{i}\right)$ be the entries of the Cartan matrix.

Choose an orientation $\Omega$ on $\Gamma$, let $\epsilon_{i j}$ denote the number of edges oriented from $i$ to $j$, and fix

$$
Q_{i j}(u, v)=(-1)^{\epsilon_{i j}}(u-v)^{c_{i j}} .
$$

We let $U_{q}(\mathfrak{g})$ denote the deformed universal enveloping algebra of $\mathfrak{g}$; that is, the associative $\mathbb{C}(q)$-algebra given by generators $E_{i}, F_{i}, K_{\xi}$ for $i$ and $\xi \in Y(\mathfrak{g})$, subject to the relations:

i) $K_{0}=1, K_{\xi} K_{\xi^{\prime}}=K_{\xi+\xi^{\prime}}$ for all $\xi, \xi^{\prime} \in Y(\mathfrak{g})$,

ii) $K_{\xi} E_{i}=q^{\alpha_{i}^{\gamma}(\xi)} E_{i} K_{\xi}$ for all $\xi \in Y(\mathfrak{g})$,

iii) $K_{\xi} F_{i}=q^{\alpha_{i}^{\vee}(\xi)} F_{i} K_{\xi}$ for all $\xi \in Y(\mathrm{~g})$,

iv) $E_{i} F_{j}-F_{j} E_{i}=\delta_{i j} \frac{\tilde{K}_{i}-\tilde{K}_{-i}}{q-q^{-1}}$, where $\tilde{K}_{ \pm i}=K_{ \pm d_{i} \alpha_{i}}$

v) For all $i \neq j$

$$
\sum_{a+b=-c_{i j}+1}(-1)^{a} E_{i}^{(a)} E_{j} E_{i}^{(b)}=0 \quad \text { and } \quad \sum_{a+b=-c_{i j}+1}(-1)^{a} F_{i}^{(a)} F_{j} F_{i}^{(b)}=0 .
$$

\section{The 2-CATEgory $\mathcal{U}$}

Our primary object of study is a 2-category categorifying the universal enveloping algebra; versions of this category have been considered by Rouquier [Roua], Khovanov and Lauda [KL10] and Cautis and Lauda [CL15]. Since recent work of Brundan [Bru] has shown that the different definitions given in these papers are equivalent, we will work with the definition given in [Roua]. For simplicity of notation, if $u_{1} \ldots u_{n}$ is the composition of $n$ 1-morphisms in a 2-category, we let $x^{(\ell)}$ for $x: u_{\ell} \rightarrow u_{\ell}$ a 2-morphism horizontal composition $1_{u_{1}} \otimes 1_{u_{2}} \otimes \cdots \otimes 1_{u_{\ell-1}} \otimes x \otimes 1_{u_{\ell+1}} \otimes \cdots \otimes 1_{u_{n}}$, and similarly with $x^{(\ell, \ell+1)}$ for $x: u_{\ell} u_{\ell+1} \rightarrow u_{\ell} u_{\ell+1}$. 
Definition 1.1 $\mathcal{U}$ is the 2-category with:

- objects given by the weight lattice;

- 1-morphisms freely generated under composition and direct sum by adjoint 1-morphisms $\mathcal{F}_{i}$ and their right adjoints $\mathcal{E}_{i}$ via the (co)unit

$$
\iota: \mathbb{1}_{\lambda} \rightarrow \mathcal{E}_{i} \mathcal{F}_{i} \mathbb{1}_{\lambda} \quad \epsilon: \mathcal{F}_{i} \mathcal{E}_{i} \mathbb{1}_{\lambda} \rightarrow \mathbb{1}_{\lambda} \quad \text { for } i \in \Gamma ;
$$

- 2-morphisms

$$
\begin{gathered}
y_{i}: \mathcal{F}_{i} \rightarrow \mathcal{F}_{i} \quad \psi_{i j}: \mathcal{F}_{i} \mathcal{F}_{j} \rightarrow \mathcal{F}_{j} \mathcal{F}_{i} \\
\xi_{i, j, \lambda}: \mathcal{E}_{i} \mathcal{F}_{j} \mathbb{1}_{\lambda} \oplus \mathbb{1}_{\lambda}^{\oplus \delta_{i j} \max \left(0,-\lambda^{i}\right)} \rightarrow \mathcal{F}_{j} \mathcal{E}_{i} \mathbb{1}_{\lambda} \oplus \mathbb{1}_{\lambda}^{\oplus \delta_{i j} \max \left(0, \lambda^{i}\right)} .
\end{gathered}
$$

These 2-morphisms are subject to the relations:

$$
\begin{aligned}
& \psi_{i j} y_{i}^{(1)}= \begin{cases}y_{i}^{(2)} \psi_{i j}+1 & \text { if } i=j, \\
y_{i}^{(2)} \psi_{i j} & \text { if } i \neq j ;\end{cases} \\
& y_{j}^{(1)} \psi_{i j}= \begin{cases}\psi_{i j} y_{j}^{(2)}+1 & \text { if } i=j, \\
\psi_{i j} y_{j}^{(2)} & \text { if } i \neq j ;\end{cases} \\
& \psi_{j i} \psi_{i j}= \begin{cases}0 & \text { if } i=j, \\
Q_{i j}\left(y_{i}^{(1)}, y_{j}^{(2)}\right) & i \neq j ;\end{cases} \\
& \psi_{j k}^{(1,2)} \psi_{i k}^{(2,3)} \psi_{i j}^{(1,2)}= \begin{cases}\psi_{i j}^{(2,3)} \psi_{i k}^{(1,2)} \psi_{j k}^{(2,3)}+\frac{Q_{i j}\left(y_{k}^{(1)}, y_{j}^{(2)}\right)-Q_{i j}\left(y_{k}^{(3)}, y_{j}^{(2)}\right)}{y_{i}^{(1)}-y_{k}^{(3)}} & \text { if } i=k, \\
\psi_{i j}^{(2,3)} \psi_{i k}^{(1,2)} \psi_{j k}^{(2,3)} & \text { otherwise, }\end{cases}
\end{aligned}
$$

Furthermore, let $\sigma_{i, j, \lambda}: \mathcal{F}_{j} \mathcal{E}_{i} \mathbb{1}_{\lambda} \rightarrow \mathcal{E}_{i} \mathcal{F}_{j} \mathbb{1}_{\lambda}$ be given by

$$
\sigma_{i, j, \lambda}=\left(1_{\varepsilon_{i} \mathcal{F}_{j}} \otimes \epsilon\right)\left(1_{\mathcal{E}_{i}} \otimes \psi_{i j} \otimes 1_{\mathcal{E}_{j}}\right)\left(\iota \otimes 1_{\mathcal{F}_{j} \varepsilon_{i}}\right) .
$$

We also have the relation

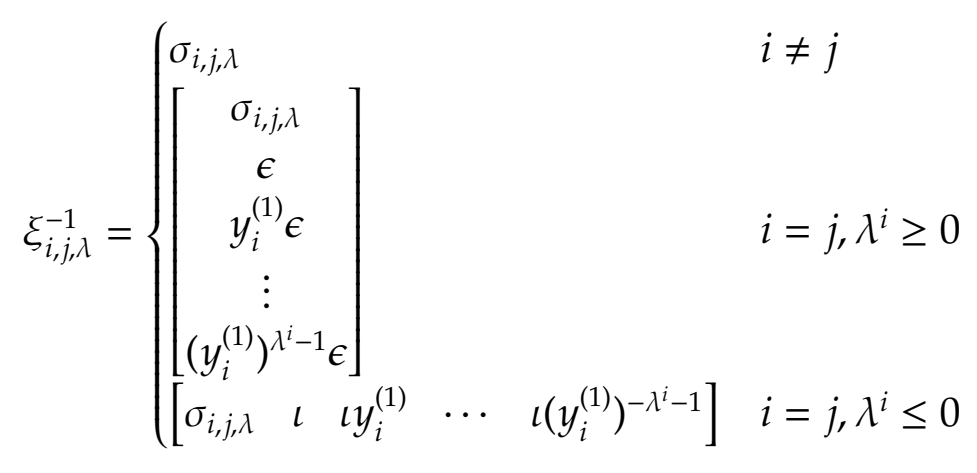

As in [KL10], we let $\mathcal{U}$ denote the 2-category where every Hom-category is replaced by its idempotent completion; we note that since every object in $\mathcal{U}$ has a finitedimensional degree 0 part of its endomorphism algebra, every Hom-category satisfies the Krull-Schmidt property. 
This 2-category is a categorification of the universal enveloping algebra is the sense that:

Theorem 1.2 ([Webc, $\epsilon .8]$ ) The graded Grothendieck group of $\dot{\mathcal{U}}$ is isomorphic to $\dot{\mathbf{U}}_{q}^{\mathbb{Z}}$, Lusztig's integral modified quantum universal enveloping algebra.

The algebra $\dot{\mathbf{U}}_{q}$ can be thought of as $U_{q}(\mathfrak{g})$ with additional idempotents $1_{\lambda}$ for integral weights $\lambda$ which satisfy the relations of projection to the $\lambda$-weight space. The integral form $\dot{\mathbf{U}}_{q}^{\mathbb{Z}}$ is generated over $\mathbb{Z}\left[q, q^{-1}\right]$ by $1_{\lambda}, E_{i} 1_{\lambda}, F_{i} 1_{\lambda}$ for all $\lambda$. The map from the Grothendieck group sends the class of the 1-morphism $\left[\mathcal{E}_{i}: \lambda \rightarrow \lambda+\alpha_{i}\right]$ to $E_{i} 1_{\lambda}$, and similarly for $\mathcal{F}_{i}$. This theorem was first conjectured by Khovanov and Lauda [KL10] and proven by them in the special case of $\mathfrak{s l}_{n}$.

\section{Quiver varieties}

Recall that $\Gamma$ denotes the Dynkin graph of $\mathfrak{g}$.

Definition 2.1 For each orientation $\Omega$ of $\Gamma$ (thought of as a subset of the edges of the oriented double), a representation of $(\Gamma, \Omega)$ with shadows is

- a pair of finite dimensional $\mathbb{C}$-vector spaces $V=\oplus_{i \in \Gamma} V_{i}$ and $W=\oplus_{i \in \Gamma} W_{i}$, graded by the vertices of $\Gamma$, and

- a map $x_{e}: V_{\omega(e)} \rightarrow V_{\alpha(e)}$ for each oriented edge (as usual, $\alpha$ and $\omega$ denote the head and tail of an oriented edge), and

- a map $z: V \rightarrow W$ that preserves grading.

We let $\mathbf{w}$ and $\mathbf{v}$ denote $\Gamma$-tuples of integers.

For now, we fix an orientation $\Omega$, though we will sometimes wish to consider the collection of all orientations. With this choice, we have the universal $(\mathbf{w}, \mathbf{v})-$ dimensional representation

$$
E_{\mathbf{v}, \mathbf{w}}=\bigoplus_{i \rightarrow j} \operatorname{Hom}\left(\mathbb{C}^{v_{i}}, \mathbb{C}^{v_{j}}\right) \oplus \bigoplus_{i} \operatorname{Hom}\left(\mathbb{C}^{v_{i}}, \mathbb{C}^{w_{i}}\right)
$$

In moduli terms, this is the moduli space of actions of the quiver (in the sense above) on the vector spaces $\mathbb{C}^{\mathbf{v}}, \mathbb{C}^{\mathbf{w}}$, with their chosen bases considered as additional structure.

If we wish to consider the moduli space of representations where $V$ has fixed graded dimension (rather than of actions on a fixed vector space), we should quotient by the group of isomorphisms of quiver representations: $G_{\mathbf{v}}=\prod_{i} \mathrm{GL}\left(\mathbb{C}^{v_{i}}\right)$ acting by pre- and post-composition. The result is the moduli stack of $\mathbf{v}$-dimensional representations shadowed by $\mathbb{C}^{\mathbf{w}}$, which we can define as the stack quotient

$$
X_{\mathbf{v}}^{\mathbf{w}}=E_{\mathbf{v}, \mathbf{w}} / G_{\mathbf{v}} \text {. }
$$

This is not a scheme in the usual sense, but rather a smooth Artin stack. Since we will only be interested in the constructible derived category of sheaves on this stack, 
we do not need the full machinery of Artin stacks, and could consider instead the equivariant derived category of $E_{\mathbf{v}, \mathbf{w}}$ as in the book of Bernstein and Lunts [BL94] or as described by the author and Williamson [WW]. We will always consider this space as having the classical topology.

By convention, if $w_{i}=\alpha_{i}^{\vee}(\lambda)$ and $\xi=\lambda-\sum v_{i} \alpha_{i}$, then $X_{\xi}^{\lambda}=X_{\mathbf{v}}^{\mathbf{w}}$ (if the difference is not in the positive cone of the root lattice, then this is by definition empty), and $X^{\lambda}=\dot{ப}_{\xi} X_{\xi}^{\lambda}$.

As mentioned in the introduction, our construction is inspired by the work of $\mathrm{Li}$ [Lia] and that of Zheng [Zhe]. Li defines a 2-category built from perverse sheaves on double framed quiver varieties.

Definition 2.2 Li's 2-category is defined as follows:

- 0-morphisms are dimension vectors for the quiver $\Gamma$,

- 1-morphisms between $\mathbf{d}$ and $\mathbf{d}^{\prime}$ are objects of geometric origin in the localized derived category which Li denotes by $\mathscr{D}^{-}\left(E_{\Omega}\left(k^{\lambda}, k^{\mathrm{d}}, k^{\mathrm{d}^{\prime}}\right)\right)$, with product given by the convolution product of [Lia, (16)].

- 2-morphisms are morphisms in the category described above.

For certain technical purposes, it is much more convenient for us to use a different 2-category built using quantizations. Let

$$
\mathfrak{M}_{\xi}^{\lambda}=T^{*} E_{\xi}^{\lambda} / / \operatorname{det} G_{\xi}=\xi^{-1}(0)^{s} / G_{\xi}
$$

be the Nakajima quiver variety attached to $\lambda$ and $\xi$; this is a smooth, quasi-projective variety which arises through geometric invariant theory as an open subset of the cotangent bundle of $X_{\xi}^{\lambda}$. See [Nak94, Nak98] for a more detailed discussion of the geometry of these varieties.

Any point in $T^{*} E_{\xi}^{\lambda}$ can be thought of as a representation of the doubled quiver of $\Gamma$ (with the framing maps also doubled). The subset $\mu^{-1}(0)$ can be thought of as parameterizing representations that descend to a certain quotient of the doubled path algebra called the preprojective algebra. A particularly important result for us is a description of the stable locus in terms of representation theory:

Lemma 2.3 ([Nak94, 3.5]) The subvariety $\mu^{-1}(0)^{s}$ is the subset whose associated preprojective representation has no non-trivial subrepresentation killed by all shadow maps.

Recall that a quantization of the variety $\mathfrak{M}_{\xi}^{\lambda}$ as defined in [BK04] or [BPW, §3] is a sheaf $\mathcal{A}_{\xi}^{\prime}$ of flat $\mathbb{C}[[h]]$-algebras with $\mathcal{A}_{\xi}^{\prime} / h \mathcal{A}_{\xi}^{\prime} \cong O_{\mathfrak{M}_{\xi}^{\lambda}}$ such the induced Poisson structure on $O_{\mathfrak{M}_{\varepsilon}^{\lambda}}$ matches the standard holomorphic symplectic structure on a quiver variety (induced from the cotangent bundle $T^{*} E_{\xi}^{\lambda}$ ). For such a quantization, we let $\mathcal{A}_{\xi}=\mathcal{A}_{\xi}^{\prime}\left[h^{-1}\right]$. 
One method of constructing such quantizations is quantum Hamiltonian reduction. This operation was introduced in an algebraic context by Crawley-Boevey, Etingof and Ginzburg [CBEG07] (though in many contexts it appeared even earlier), and in the geometric form of interest to us in [KR08, 2.8(i)]. Throughout we'll follow the conventions of [BPW] for Hamiltonian reduction of quantizations and refer the reader to constructions there (even those which have appeared in older papers) in the interest of consistency. We let $\mathcal{R}^{\prime}$ be the sheaf of microlocal differential operators on $T^{*} E_{\xi}^{\lambda}$, that is, the Rees algebra for the usual order filtration of the sheaf $\mathcal{D}_{E_{\xi}^{\lambda}}$ of differential operators sheafified over $T^{*} E_{\xi}^{\lambda}$. This algebra is naturally a quantization of $T^{*} E_{\xi}^{\lambda}$ (see [BPW, §4.1]). We let $\mathcal{R}=\mathcal{R}^{\prime}\left[h^{-1}\right]$; this is a sheaf on $T^{*} E_{\xi}^{\lambda}$ such that taking pushforward under $\pi: T^{*} E_{\xi}^{\lambda}$ we obtain $\pi_{*} \mathcal{R} \cong \mathcal{D}_{E_{\xi}^{\lambda}}((h))$. In particular,

$$
\Gamma\left(T^{*} E_{\xi}^{\lambda} ; \mathcal{R}\right) \cong \Gamma\left(E_{\xi}^{\lambda} ; \mathcal{D}_{E_{\xi}^{\lambda}}\right)((h)) .
$$

Differentiating the action of $G$ on $E_{\xi}^{\lambda}$ induces a Lie algebra map from $\mathfrak{g}$ to vector fields on $E_{\xi}^{\lambda}$, and thus a non-commutative moment map $m: U(\mathfrak{g}) \rightarrow \mathcal{R}$. Let $\mathcal{R}_{\mathcal{S}}^{\prime}, \mathcal{R}_{\mathcal{S}}$ be the pullback of these sheaves to the stable locus $\mathcal{S}$. As in [BPW, §3.4], we let

$$
\mathcal{E}=\mathcal{R} / \mathcal{R} m(\mathfrak{g}) \quad \mathcal{E}_{\mathcal{S}}=\mathcal{R}_{\mathcal{S}} / \mathcal{R}_{\mathcal{S}} m(\mathfrak{g})
$$

and consider the endomorphism sheaf $\mathcal{E} n d_{\mathcal{R}_{\mathcal{S}}}\left(\mathcal{E}_{\mathcal{S}}\right)$, which is naturally supported on $\mu^{-1}(0)^{s}$. Let $p: \mu^{-1}(0)^{s} \rightarrow \mathfrak{M}_{\xi}^{\lambda}$ be the quotient map.

Definition 2.4 We let $\mathcal{A}_{\xi}:=p_{*} \mathcal{E} n d_{\mathcal{R}_{\mathcal{S}}}\left(\mathcal{E}_{\mathcal{S}}\right)$, the pushforward sheaf on $\mathfrak{M}_{\xi}^{\lambda}$.

We actually have that $\mathcal{A}_{\xi}=\mathcal{A}_{\xi}^{\prime}\left[h^{-1}\right]$ for a quantization $\mathcal{A}_{\xi}^{\prime}$ obtained from $\mathcal{R}^{\prime}$ by a similar reduction procedure as in [BPW, §3.4]. The quantizations of $\mathfrak{M}_{\xi}^{\lambda}$ can be classified by a cohomological invariant called its period. This is a class in $h H^{2}\left(\mathfrak{M}_{\xi}^{\lambda} ; \mathbb{C}\right)[[h]]$ and any such class can be realized by a quantization since $H^{2}\left(\mathfrak{M}_{\xi}^{\lambda} ; \mathbb{C}\right)=H^{1,1}\left(\mathfrak{M}_{\xi}^{\lambda} ; \mathbb{C}\right)$.

On $X_{\mathrm{v}}^{\mathbf{w}}$, we have a tautological vector bundle $\mathscr{V} i$ whose fiber over a representation is $V_{i}$, the part of that representation at node $i$; let $\mathscr{L}_{i}=\operatorname{det}\left(\mathscr{V}_{i}\right)$. By [BPW] 6.4], the period of $\mathcal{A}_{\xi}^{\prime}$ is

$$
1 / 2 \sum_{i \in \Gamma}\left(w_{i}+\sum_{j \rightarrow i} v_{j}-\sum_{i \rightarrow j} v_{j}\right) c_{1}\left(\mathscr{L}_{i}\right) h
$$

Note that this period depends on the choice of orientation of $\Gamma$, but its class modulo $h H^{2}\left(\mathfrak{M}_{\xi}^{\lambda} ; \mathbb{Z}\right)$ does not. Also, this is not always an integral class; this is a generalization of the fact that differential operators, thought of as a quantization of a cotangent bundle, do not always have integral period (as [BPW] 3.10] shows). If, as suggested in the introduction, we think of the quiver variety as the cotangent bundle of a hypothetical space $Y$, this would be the algebra of untwisted differential operators on $Y$, and the quantization with period 0 would be the differential operators in the square root of the canonical bundle of $Y$. 
The quiver varieties carry a natural $\mathbb{C}^{*}$-action inherited from the action on $T^{*} E_{\mathbf{v}, \mathbf{w}}$ scaling the cotangent fibers. The sheaf of algebras $\mathcal{A}_{\xi}$ carries a equivariant structure over $\mathbb{C}^{*}$ (see [Los12, 2.3.3]). We let $\mathcal{A}_{\xi}$-mod denote the category of $\mathbb{C}^{*}$-equivariant good modules over $\mathcal{A}_{\xi}$ (as defined in [BPW, §4]).

The Hamiltonian reduction realization of $\mathcal{A}_{\xi}$ gives us a functor $\mathfrak{r}: \mathcal{D}_{X_{\xi}^{\lambda}}$-mod $\rightarrow$ $\mathcal{A}_{\xi}$-mod (called the "Kirwan functor" in [BPW, \$5.4], where this functor is studied extensively) from $D$-modules on $X_{\xi}^{\lambda}$ to $\mathcal{A}_{\xi}$-modules. This functor proceeds by replacing a D-module $\mathcal{M}$ by its microlocalization $\underline{\mu} \mathcal{M}:=\mathcal{R} \otimes_{\pi^{-1} \mathcal{D}_{X_{\xi}^{\lambda}}} \pi^{-1} \mathcal{M}$, which is a sheaf on $T^{*} X_{\xi}^{\lambda} \cong \mu^{-1}(0) / G$, and then restricting to $\mathfrak{M}_{\mu}^{\lambda} \subset T^{*} X_{\xi}^{\lambda}$. That is:

Definition 2.5 The Kirwan functor is the restriction $\mathfrak{r}(\mathcal{M})=\left.\mathcal{M}\right|_{\mathfrak{M}_{\xi}^{\lambda}}$.

While using stack language is elegant, one can also describe this in terms of the associated $G_{\xi}$-equivariant D-module $\mathcal{M}^{\prime}$ on $E_{\xi}^{\lambda}$; this has microlocalization $\mu \mathcal{M}^{\prime}$ supported on $\mu^{-1}(0)$ by equivariance. We restrict this to the stable locus, and take the invariant pushforward $\mathfrak{r}(\mathcal{M})=p_{*} \mathcal{H o m}_{\mathcal{R}_{\mathcal{S}}}\left(\mathcal{E}_{\mathcal{S}},\left.\underline{\mu} \mathcal{M}^{\prime}\right|_{\mathcal{S}}\right)$, with its natural $\mathcal{A}_{\xi}$-action. Since on $\mu^{-1}(0)^{s}$, the $G_{\xi}$ action is free, we always stay within the world of varieties.

Perhaps the most important property for us is that:

Proposition 2.6 ([BPW, 5.17], [MN, 1.1]) The functor $\mathfrak{r}$ admits left and right adjoints

$$
\mathfrak{r}_{!}: \mathcal{A}_{\xi}-\bmod \rightarrow \mathcal{D}_{X_{\xi}^{\lambda}}-\bmod \quad \mathfrak{r}_{*}: \mathcal{A}_{\xi}-\bmod \rightarrow \mathcal{D}_{X_{\xi}^{\lambda}}-\bmod ,
$$

such that $\mathfrak{r} \circ \mathfrak{r}_{!} \cong \mathfrak{r} \circ \mathfrak{r}_{*} \cong \mathrm{id}$.

Definition 2.7 We let $Q^{\lambda}$ be the 2-category where

- 0 -morphisms are dimension vectors for the quiver $\Gamma$,

- 1-morphisms between $\mathbf{d}$ and $\mathbf{d}^{\prime}$ given by the bounded-below derived category of complexes of modules over $\mathcal{A}_{\xi} \otimes \mathcal{A}_{\xi^{\prime}}^{o p}$. Composition of 1-morphisms $\mathcal{H}_{1}: \mathfrak{M}_{\xi_{1}}^{\lambda} \rightarrow \mathfrak{M}_{\xi_{2}}^{\lambda}$ and $\mathcal{H}_{2}: \mathfrak{M}_{\xi_{2}}^{\lambda} \rightarrow \mathfrak{M}_{\xi_{3}}^{\lambda}$ is given by convolution

$$
\mathcal{H}_{1} \star \mathcal{H}_{2}:=\left(p_{13}\right)_{*}\left(p_{12}^{*} \mathcal{H}_{1} \stackrel{L}{\otimes}_{\mathcal{A}_{\xi_{2}}} p_{23}^{*} \mathcal{H}_{2}\right)\left[-\operatorname{dim}\left(\mathfrak{M}_{\xi_{1}}^{\lambda} \times \mathfrak{M}_{\xi_{3}}^{\lambda}\right)\right]
$$

- 2-morphisms are morphisms in the category described above; we consider this as a graded category with the homological grading.

This 2-category receives a natural 2-functor from the analytic version of Li's 2category; the (classical topology) derived category of $X_{\xi}^{\lambda}$ has a functor to the derived category of $\mathcal{D}_{X_{\xi}^{\lambda}}$-modules given by the Riemann-Hilbert correspondence, and the functor $r$ kills the necessary subcategories to induce a 2-functor from the localization. In order to confirm that this is a 2-functor, we would have check that we could also define convolution as in Li's category [Lia, §4.8] (though, his definition is "dual" to ours, since he uses the left, rather than right adjoint of reduction). 


\section{HeCKe CORRESPONDENCES AND CATEgORICAL ACtions}

We let $X_{\xi ; \nu}^{\lambda}$ denote the moduli stack of short exact sequences ("Hecke correspondences") where the subobject belongs in $X_{\xi}^{\lambda}$, the total object in $X_{\xi-v}^{\lambda}$ and the quotient in $X_{-v}^{0}$.

This moduli stack is naturally equipped with projections

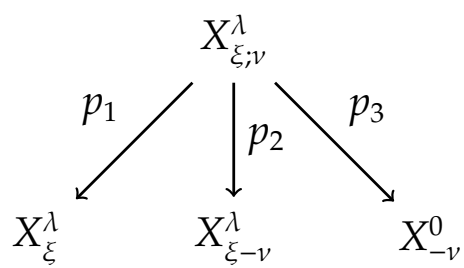

which we can think of more abstractly as taking the subobject, total object and quotient, respectively.

If $\lambda-\xi=\sum v_{i}^{\prime} \alpha_{i}$ and $v=\sum v_{i}^{\prime \prime} \alpha_{i}$, then we can also think of this space more concretely. We let

$$
E_{\xi ; v}^{\lambda} \cong \bigoplus_{i \rightarrow j} \operatorname{Hom}\left(\mathbb{C}^{v_{i}^{\prime}}, \mathbb{C}^{v_{j}^{\prime}}\right) \oplus \operatorname{Hom}\left(\mathbb{C}^{v_{i}^{\prime \prime}}, \mathbb{C}^{v_{j}^{\prime}}\right) \oplus \operatorname{Hom}\left(\mathbb{C}^{v_{i}^{\prime \prime}}, \mathbb{C}^{v_{j}^{\prime \prime}}\right) \oplus \bigoplus_{i} \operatorname{Hom}\left(\mathbb{C}^{v_{i}}, \mathbb{C}^{w_{i}}\right)
$$

and let $P_{\xi ; v}$ be the parabolic in $G_{\mathrm{v}}$ which preserves $\mathbb{C}^{v_{i}^{\prime}}$ inside of $\mathbb{C}^{v_{i}}=\mathbb{C}^{v_{i}^{\prime}} \oplus \mathbb{C}^{v_{i}^{\prime \prime}}$. We can alternatively define $X_{\xi ; v}^{\lambda}:=E_{\xi ; v}^{\lambda} / P_{\xi ; v}$.

The projection maps are also easily understood from this perspective.

- The map $\pi_{1}$ is induced by the map $E_{\xi ; v}^{\lambda} \rightarrow E_{\xi}^{\lambda}$ restricting each map to the subspace $\mathbb{C}^{v_{i}^{\prime}}$ over each node.

- The map $\pi_{2}$ is induced by the inclusion $E_{\xi ; v}^{\lambda} \hookrightarrow E_{\xi-v}^{\lambda}$ induced by the isomorphism $\mathbb{C}^{v_{i}}=\mathbb{C}^{v_{i}^{\prime}} \oplus \mathbb{C}^{v_{i}^{\prime \prime}}$.

- The map $\pi_{3}$ is induced by the map $E_{\xi ; v}^{\lambda} \rightarrow E_{-v}^{0}$ which projects to $\operatorname{Hom}\left(\mathbb{C}_{i}^{v^{\prime \prime}}, \mathbb{C}^{v_{j}^{\prime \prime}}\right)$ for each arrow $i \rightarrow j$.

These maps are compatible with group homomorphisms from $P_{\xi ; v}$ to $G_{\mathbf{v}^{\prime}}, G_{\mathbf{v}}, G_{\mathbf{v}^{\prime \prime}}$, and thus induce maps of the appropriate quotient stacks.

For each $\lambda, \xi, i$, we let

$$
\begin{array}{cc}
\tilde{\mathscr{F}}_{i}=\omega_{X_{\xi-\alpha_{i}}^{\lambda}} \otimes_{O_{X_{\xi-\alpha_{i}}^{\lambda}}}\left(p_{1} \times p_{2}\right)_{*} O_{X_{\xi ; \alpha_{i}}^{\lambda}} & \mathscr{F}_{i}=\mathfrak{r}\left(\tilde{\mathscr{F}}_{i}\right) \\
\tilde{\mathscr{E}}_{i}=\omega_{X_{\xi}^{\lambda}} \otimes_{O_{X_{\xi}^{\lambda}}}\left(p_{2} \times p_{1}\right)_{*} O_{X_{\xi ; \alpha_{i}}^{\lambda}} & \mathscr{E}_{i}=\mathfrak{r}\left(\tilde{\mathscr{E}}_{i}\right)
\end{array}
$$

Naturally, $\mathscr{F}_{i}$ is a module over $\mathcal{A}_{\xi} \otimes \mathcal{A}_{\xi-\alpha_{i}}^{o p}$ and $\mathscr{E}_{i}$ is a module over $\mathcal{A}_{\xi-\alpha_{i}} \otimes \mathcal{A}_{\xi}^{o p}$. That is, by definition, these are 1-morphisms in $Q^{\lambda}$ between the appropriate dimension vectors. They are the images under the Riemann-Hilbert correspondence of the similarly named objects in Li's development of the theory. We now proceed to our principal result: 
Theorem 3.1 We have a 2-functor of graded categories $\mathcal{G}_{\lambda}: \mathcal{U} \rightarrow Q^{\lambda}$ sending $\mathcal{E}_{i} \mapsto \mathscr{E}_{i}$ and $\mathcal{F}_{i} \mapsto \mathscr{F}_{i}$.

For now, we postpone the proof of this theorem to Section 4 , and instead discuss its variations and consequences in a bit more detail.

3.1. The non-integral case. The existence of the objects $\mathscr{E}_{i}$ and $\mathscr{F}_{i}$ depends very strongly on the fact that we use untwisted D-modules here. Consider twists

$$
\chi_{1}=\sum_{j \in \Gamma} a_{i} c_{1}\left(\mathscr{L}_{j}\right) \in H^{2}\left(X_{\xi}^{\lambda}\right) \quad \chi_{2}=\sum_{j \in \Gamma} b_{i} c_{1}\left(\mathscr{L}_{j}^{\prime}\right) \in H^{2}\left(X_{\xi-\alpha_{i}}^{\lambda}\right)
$$

on the respective varieties, where we use $\mathscr{L}_{j}^{\prime}, \mathscr{V}_{j}^{\prime}$ to denote the tautological bundles on $X_{\xi-\alpha_{i}}^{\lambda}$.

Proposition 3.2 There exists a line bundle $\mathscr{L}$ on $X_{\xi ; \alpha_{i}}^{\lambda}$ such that

$$
\begin{aligned}
& \tilde{\mathscr{F}}_{i}^{\mathscr{L}}=\omega_{X_{\xi-\alpha_{i}}^{\lambda}} \otimes_{O_{X_{\xi-\alpha_{i}}^{\lambda}}}\left(p_{1} \times p_{2}\right)_{*} \mathscr{L} \\
& \tilde{\mathscr{E}}_{i}^{\mathscr{L}}=\omega_{X_{\xi}^{\lambda}} \otimes_{O_{X_{\xi}^{\lambda}}}\left(p_{2} \times p_{1}\right)_{*} \mathscr{L}^{-1}
\end{aligned}
$$

are bimodules over $\mathcal{D}_{X_{\xi}^{\lambda}}\left(\chi_{1}\right)$ and $\mathcal{D}_{X_{\xi-\alpha_{i}}^{\lambda}}\left(\chi_{2}\right)$ if and only if $a_{i}, b_{i}, a_{j}-b_{j} \in \mathbb{Z}$ for all $j \in \Gamma$.

Proof. First we note that $X_{\xi}^{\lambda}, X_{\xi-\alpha_{i}}^{\lambda}$ and $X_{\xi ; \alpha_{i}}^{\lambda}$ are each the quotient of an affine space by an affine algebraic group. These groups are $G_{\xi}$ and $G_{\xi-\alpha_{i}}$ and a maximal parabolic in the latter, respectively. Thus the Picard groups of these spaces are naturally identified with the character group of the group in question. In practice, this means that

- $\left\{c_{1}\left(\mathscr{L}_{j}\right)\right\}_{j \in \Gamma}$ is a basis of $H^{2}\left(X_{\xi}^{\lambda} ; \mathbb{Z}\right)$,

- $\left\{c_{1}\left(\mathscr{L}_{j}^{\prime}\right)\right\}_{j \in \Gamma}$ is a basis of $H^{2}\left(X_{\xi-\alpha_{i}}^{\lambda} ; \mathbb{Z}\right)$ and

- $\left\{c_{1}\left(p_{1}^{*} \mathscr{L}_{j}\right)\right\}_{j \in \Gamma} \cup\left\{c_{1}\left(p_{2}^{*} \mathscr{L}_{i}^{\prime}\right)\right\}$ for $H^{2}\left(X_{\xi ; \alpha_{i}}^{\lambda} ; \mathbb{Z}\right)$.

In order to have the desired left and right twisted D-module structure, we must have that $c_{1}(\mathscr{L})=p_{1}^{*} \chi_{1}-p_{2}^{*} \chi_{2} ;$ by the identification of the Picard group with homology, such an $\mathscr{L}$ exists if and only if $p_{1}^{*} \chi_{1}-p_{2}^{*} \chi_{2} \in H^{2}\left(X_{\xi ; \alpha_{i}}^{\lambda} ; \mathbb{Z}\right)$.

For $j \neq i$, we have that $p_{1}^{*} c_{1}\left(\mathscr{L}_{j}\right)=p_{2}^{*} c_{1}\left(\mathscr{L}_{j}^{\prime}\right)$, but for $i$, these are independent classes. Thus, we have that

$$
p_{1}^{*} \chi_{1}-p_{2}^{*} \chi_{2}=a_{i} p_{1}^{*} c_{1}\left(\mathscr{L}_{i}\right)-b_{i} p_{2}^{*} c_{1}\left(\mathscr{L}_{i}^{\prime}\right)+\sum_{j \neq i}\left(a_{j}-b_{j}\right) p_{1}^{*} c_{1}\left(\mathscr{L}_{j}\right),
$$

which is integral if and only if $a_{i}, b_{i}, a_{j}-b_{j} \in \mathbb{Z}$.

Thus more generally, using Proposition [3.2, we can define such an action where we choose any quantization corresponding to differential operators in a line bundle on each $X_{\xi}^{\lambda}$, not just the particular one we have fixed. If we instead choose a not necessarily integral twist $\chi=\sum a_{i} c_{1}\left(\mathscr{L}_{i}\right)$, we only know at the moment how to construct 
a categorical action of the smaller Lie algebra generated by the simple root spaces where $a_{i}$ is integral.

This observation is particularly interesting in the case where $\mathfrak{g}$ is affine, $\lambda$ is the basic fundamental weight and $\xi=n \delta$. In this case, the $\mathbb{C}^{*}$-invariant section algebra $\Gamma\left(\mathfrak{M}_{\xi}^{\lambda} ; \mathcal{A}_{\xi}\right)^{\mathbb{C}^{*}}$ is a spherical symplectic reflection algebra for $S_{n}$ wr $\gamma$, where $\gamma$ matches $\mathfrak{g}$ under the Mackay correspondence by [EGGO07, Gor06, Los12]. This phenomenon of functors associated to roots appearing when particular functions on the parameter space are integral is quite suggestive in connection with Etingof's conjecture relating finite dimensional modules for these symplectic reflection algebras to affine Lie algebras [Eti12]. In fact, since the first version of this paper appeared as a preprint, Bezrukavnikov and Losev $[\mathrm{BL}]$ have proven this theorem using the functors that arise this way.

3.2. Harish-Chandra and core modules. The 2-functor $\mathcal{G}_{\lambda}$ actually lands in a much smaller subcategory of $Q^{\lambda}$. In the product $\mathfrak{M}_{\xi}^{\lambda} \times \mathfrak{M}_{\xi^{\prime}}^{\lambda}$ we still have a notion of "diagonal." By [Nak98, 3.27], the affinization of a quiver variety $\mathfrak{N}_{\xi}^{\lambda}$ lies in the moduli space of semisimple representations of the pre-projective algebra of a given dimension. We say a pair of such representations lies in the stable diagonal if they become isomorphic after the addition of trivial representations (that is, they are isomorphic up to stabilization). In more concrete terms, the global functions on $\mathfrak{N}_{\xi}^{\lambda}$ are generated by trace of the composition of the maps along a path in the doubled Crawley-Boevey quiver ${ }^{2}$ of $\Gamma$. We can define the stable diagonal to be the pairs in $\mathfrak{N}_{\xi}^{\lambda} \times \mathfrak{N}_{\xi^{\prime}}^{\lambda}$ where these traces agree for any path.

Following Nakajima, we let $Z$ denote the preimage of the stable diagonal in $\mathfrak{M}^{\lambda} \times \mathfrak{M}^{\lambda}$; this can also be thought of as the points where all the traces of loops coincide. In [BPW, §6.1], Braden, Proudfoot and the author define a 2-subcategory $\mathbf{H C}^{g}(\lambda)$ of good sheaves of $\mathcal{A}_{\xi} \otimes \mathcal{A}_{\xi}$-modules called Harish-Chandra bimodules. This is the category of modules $\mathcal{M}$ such that:

- the support of $\mathcal{M}$ is contained in $\mathrm{Z}$.

- there is a $\mathcal{A}_{\xi}^{\prime} \otimes \mathcal{A}_{\xi^{\prime}}^{\prime}$-lattice $\mathcal{M}^{\prime} \subset \mathcal{M}$ such that any global function vanishing on the stable diagonal kills the coherent sheaf $\mathcal{M}^{\prime} / h \mathcal{M}^{\prime}$. This is a condition which should be thought of as an analogue of regularity of D-modules.

Proposition 3.3 The image of $\mathcal{G}_{\lambda}$ lies in the 2-category $\mathbf{H C}^{g}(\lambda)$.

Proof. Since $\mathbf{H C}^{g}(\lambda)$ is closed under convolution, we need only check these conditions for $\mathscr{E}_{i}$ and $\mathscr{F}_{i}$. We have already checked that the supports of these modules are Hecke correspondences, and thus lie in $Z$.

\footnotetext{
${ }^{2}$ The Crawley-Boevey quiver is $\Gamma$ with an additional vertex $\infty$ and $w_{i}$ new edges attaching $i$ to $\infty$. We can think of an element of $E_{\xi}^{\lambda}$ as a representation of this quiver with $\mathbb{C}$ placed on $\infty$, and thinking of each row in the matrix of the map $\mathbb{C}^{v_{i}} \rightarrow \mathbb{C}^{w_{i}}$ as the map along a different edge.
} 
Furthermore, the D-modules $\tilde{\mathscr{E}}_{i}$ and $\tilde{\mathscr{F}}_{i}$ are the pushforwards of regular D-modules, and thus themselves regular. The corresponding very good filtrations on these Dmodules have associated graded killed by any global function which vanishes on their support. Note that global functions on $\mathfrak{M}_{\xi}^{\lambda} \times \mathfrak{M}_{\xi \pm \alpha_{i}}^{\lambda}$ are the same as invariant functions on $E_{\xi}^{\lambda} \times E_{\xi \pm \alpha_{i}}^{\lambda}$. Since any invariant function whose reduction vanishes on the stable diagonal must vanish on the support of $\tilde{\mathscr{E}}_{i}$ and $\tilde{\mathscr{F}}_{i}$, it acts trivially on their associated graded. Thus, it also acts trivially on the induced lattice on $\mathscr{E}_{i}$ or $\mathscr{F}_{i}$, and we are done.

This draws an analogy between the categorical action $\mathcal{G}_{\lambda}$ and the action of the monoidal category of Harish-Chandra bimodules (in the classical sense) on various categories of representations of $\mathfrak{g}$. The latter is a categorification of the Hecke algebra, which has

- its original representation-theoretic description,

- a geometric one via the localization theorem of Beilinson and Bernstein [BB81], and

- a diagrammatic description in the guise of Soergel bimodules given by the work of Elias and Khovanov in type A [EK10] and work of Elias and Williamson in general [EW].

The 2-category $\mathcal{U}$ was first defined in a purely diagrammatic manner, so it is striking evidence of its naturality (at least to the author) to see it arise in a geometric context as well.

This observation also has applications in practice. Consider a system of subvarieties $J_{\xi} \subset \mathfrak{M}_{\xi}^{\lambda}$ which is closed under convolution with $Z$. Since the support of the convolution of two modules is contained in the convolution of their supports, the category of modules supported on $J_{\xi}$ is closed under this categorical action. Examples include:

- the cores $L_{\xi}^{\lambda}$ of the varieties $\mathfrak{M}_{\xi}^{\lambda}$; that is, the subvariety of representations which are nilpotent as representations of the preprojective algebra. Alternatively, the core $L_{\xi}^{\lambda}$ is the preimage of the unique fixed point of the conic $\mathbb{C}^{*}$-action on $\mathfrak{N}_{\xi}^{\lambda}$.

A sheaf of $\mathcal{A}_{\xi}$-modules is called a core module if it is supported on the core. Let $C_{\xi}^{\lambda}$ be the category of core modules on $\mathfrak{M}_{\xi}^{\lambda}$ for our fixed quantization $\mathcal{A}_{\xi}$, and $C^{\lambda}:=\oplus_{\xi} C_{\xi}^{\lambda}$.

- the points attracted to the core under a $\mathbb{C}^{*}$-action for which the symplectic form has positive weight. The modules supported on these subvarieties (subject to a regularity condition like $\mathbf{H C}^{g}$ ) are an analogue of category $O$ and are studied in much greater detail by Braden, Licata, Proudfoot and the author in [BLPW].

Thus, we have that:

Corollary 3.4 The sum $C^{\lambda}$ carries a categorical g-action. 
In fact, we can prove something stronger here. Recent work of Baranovsky and Ginzburg $[\overline{B G}]$ shows that number of simples in $C_{\xi}^{\lambda}$ is less than or equal to the dimension of cohomology group $H^{\text {mid }}\left(\mathfrak{M}_{\xi}^{\lambda} ; \mathbb{C}\right)$. By work of Nakajima [Nak98], $\operatorname{dim} H^{\text {mid }}\left(\mathfrak{M}_{\xi}^{\lambda} ; \mathbb{C}\right)=$ $\operatorname{dim}\left(V_{\lambda}\right)_{\xi}$, the weight multiplicity of $\xi$ in the simple g-representation $V_{\lambda}$ with highest weight $\lambda$. Let $K\left(C^{\lambda}\right)$ be the Grothendieck group of the abelian category of $\mathbb{C}^{*}$ equivariant good core modules.

Theorem 3.5 There is an isomorphism of $\mathfrak{g}$-representations $K\left(C^{\lambda}\right) \cong V_{\lambda}$.

We should emphasize that this is only true in the case where the quantization is integral (it corresponds to D-modules on an honest line bundle). There is always an injective map $K\left(C^{\lambda}\right) \rightarrow V_{\lambda}$ given by characteristic cycles (see [BPW, 6.2] or [KS]), but outside of the integral case, it seems to never to be surjective. Recent work of Bezrukavnikov and Losev [BL] has calculated the structure of this Grothendieck group in non-integral cases for finite type, and certain especially important affine cases.

Proof. The space $K\left(C^{\lambda}\right)$ is an g-representation, which has a weight decomposition by the definition of a categorical $g$-action. Furthermore, by the result from $[\overline{B G}]$ referenced above, the weight multiplicities of this representation are no more than those of the simple $V_{\lambda}$.

On the other hand $\mathcal{A}_{\lambda} \cong \mathbb{C}$, thought of as a sheaf on a point. Thus, $\mathcal{C}_{\lambda}^{\lambda}$ is equivalent to the category of $\mathbb{C}$-vector spaces, so $K\left(C^{\lambda}\right)$ has weight multiplicity 1 for $\lambda$. By our bound by $\operatorname{dim}\left(V_{\lambda}\right)_{\xi}$, the weight $\lambda$ is maximal among weights with non-zero multiplicity, since any higher weight corresponds to an empty quiver variety. Thus all vectors of weight $\lambda$ in $K\left(C^{\lambda}\right)$ are highest weight vectors.

This shows that $V_{\lambda}$ occurs as a composition factor with multiplicity 1 , and by the bound on weight multiplicity, there can be no others.

One of the powerful aspects of categorical actions is that they constrain the structure of a category. In particular, categorifications of simple representations are essentially unique, by work of Rouquier [Roua]. Attached to the weights $\lambda$ and $\xi$, there is an algebra $R_{\xi}^{\lambda}$, the cyclotomic KLR algebra, such that the projective modules over this algebra categorify the simple representation $V_{\lambda}$. By this uniqueness result, we have the following:

Theorem 3.6 There is a semi-simple core module $C_{\xi}$ for each $\xi$ such that $\operatorname{Ext}^{\bullet}\left(C_{\xi}, C_{\xi}\right) \cong$ $R_{\xi}^{\lambda}$.

In fact in [Webe], we will show that the cohomology of $\operatorname{Ext}^{\bullet}\left(C_{\xi}, C_{\xi}\right)$ is formal, so Morita theory for dg-categories will imply that $\operatorname{Ext}^{\bullet}\left(C_{\xi},-\right)$ induces an equivalence of dg-categories $R_{\xi}^{\lambda}$-dgmod $\cong D^{b}\left(C_{\xi}^{\lambda}\right)$. 
Proof. The desired module is the sum

$$
C_{\xi}=\bigoplus_{\mathbf{i}} \mathcal{F}_{i_{1}} \ldots \mathcal{F}_{i_{n}} \mathcal{A}_{\lambda}^{\lambda}
$$

for $\mathbf{i}=\left(i_{1}, \ldots, i_{n}\right)$ the set of all sequences such that $\xi+\alpha_{i_{1}}+\cdots+\alpha_{i_{n}}=\lambda$. This is the Hamiltonian reduction of the D-module $\oplus_{\mathbf{i}} \tilde{\mathscr{F}}_{i_{1}} \star \cdots \star \tilde{\mathscr{F}}_{i_{n}} \star O_{\lambda}^{\lambda}$ (where $O_{\lambda}^{\lambda}$ is the structure sheaf of the point $E_{\lambda}^{\lambda}$ ). This D-module is semi-simple by the Decomposition Theorem, so the same is true of its reduction.

By [Webd, 2.38], we have an isomorphism of the deformed cyclotomic quotient $\check{R}_{\xi}^{\lambda} \otimes_{\check{R}_{\lambda}^{\lambda}} \operatorname{Ext}^{\bullet}\left(C_{\lambda}, C_{\lambda}\right) \rightarrow \operatorname{Ext}{ }^{\bullet}\left(C_{\xi}, C_{\xi}\right)$. Since $C_{\lambda}=\mathcal{A}_{\lambda}^{\lambda}$, this tensor product is just $R_{\xi}^{\lambda}$, and we have the desired isomorphism $\operatorname{Ext}^{\bullet}\left(C_{\xi}\right) \cong R_{\xi}^{\lambda}$.

Thus the non-isomorphic simple summands of $C_{\xi}$ are in bijection with indecomposable projectives $R_{\xi}^{\lambda}$. We know from [Webd, 2.29] that the K-group of $R_{\xi}^{\lambda}$ has dimension given by the weight multiplicity of $\xi$ in $V_{\lambda}$, so this is the number of non-isomorphic simple summands of $C_{\xi}$. By the upper bound of Baranovsky and Ginzburg, every core simple must be a summand of this module.

Note that this equivalence matches the indecomposable projective modules over $R_{\xi}^{\lambda}$ to the simple modules in $C_{\xi}^{\lambda}$. Since the main result of [VV11] matches these indecomposables with Lusztig's canonical basis, we have that:

Corollary 3.7 The isomorphism $K^{0}\left(C_{\xi}^{\lambda}\right) \cong V_{\lambda}$ matches the classes of simples and Lusztig's canonical basis.

In particular, this shows that cyclotomic KLR algebras and Lusztig's canonical basis for a simple have a natural geometric origin based on quiver varieties. Core modules may not seem like a familiar object to most readers, but they are closely linked to finite dimensional modules over the section algebra $A_{\xi}=\Gamma\left(\mathfrak{M}_{\xi}^{\lambda} ; \mathcal{A}_{\xi}\right)^{\mathbb{C}^{*}}$. It follows from [BPW, Th. B.1] that:

Theorem 3.8 Every core module $\mathcal{M}$ has a finite dimensional space of $\mathbb{C}^{*}$-invariant sections $\Gamma\left(\mathfrak{M}_{\xi}^{\lambda} ; \mathcal{M}\right)^{\mathbb{C}^{*}}$. Furthermore, there exist choices of integral period such that $\Gamma\left(\mathfrak{M}_{\xi}^{\lambda} ;-\right)^{\mathbb{C}^{*}}$ is an equivalence of categories between core modules and finite dimensional $A_{\xi}$-modules.

Even when this sections functor fails to be an equivalence, it is often a derived equivalence; the set of such periods actually contains a Zariski open set. The paper [BPW] contains a much more detailed discussion of when localization and derived localization hold.

While it does not follow from such a simple uniqueness argument, one can generalize Theorem 3.6 to one connecting category $O^{\prime}$ s to the weighted KLR algebras introduced in [Webf]. This is proven in in [Webe, Th. A]. 
3.3. Canonical bases. In this subsection, we assume that $\Gamma$ is an ADE Dynkin diagram. Another canonical basis worth considering is that for the modified quantum universal enveloping algebra $\dot{\mathbf{U}}$. By [Web15, Th. A], this canonical basis coincides with the classes of the indecomposable 1-morphisms in $\mathcal{U}$.

Lemma 3.9 The 2-functor $\mathcal{G}_{\lambda}$ is full on 2-morphisms, that is for any 1-morphisms $u$ and $v$, the $\operatorname{map} \operatorname{Hom}_{\mathcal{U}}(u, v) \rightarrow \operatorname{Hom}_{Q_{\lambda}}\left(\mathcal{G}_{\lambda}(u), \mathcal{G}_{\lambda}(v)\right)$.

Proof. We induct downward on the usual order on the weight lattice generated by $\mu-\alpha_{i}<\mu$.

We have that $\mathfrak{M}_{\lambda}^{\lambda}$ is a point, so the only non-trivial 1-morphism is the identity, and its endomorphisms are just the scalars. In this case, fullness is clear. This establishes the base case.

Assume that we know the theorem for 1-morphisms $\mu^{\prime} \rightarrow v^{\prime}$ where either $\mu^{\prime}>\mu$ or $v^{\prime}>v$. Assume that $u$ and $v$ are indecomposable. Recall that $\mathcal{U}$ has a "triangular decomposition" into two subcategories $\mathcal{U}^{+}$and $\mathcal{U}^{-}$generated by the $\mathcal{E}_{i}{ }^{\prime} \mathrm{s}$ and $\mathcal{F}_{i}$ 's respectively. We now prove two smaller claims:

(1) if $v$ is not in the image of $\mathcal{U}^{-}$, then $\operatorname{Hom}_{\mathcal{U}}(u, v) \rightarrow \operatorname{Hom}_{\mathcal{Q}_{\lambda}}\left(\mathcal{G}_{\lambda} u, \mathcal{G}_{\lambda} v\right)$.

(2) if $u$ is not in the image of $\mathcal{U}^{+}$, then $\operatorname{Hom}_{\mathcal{U}}(u, v) \rightarrow \operatorname{Hom}_{2_{\lambda}}\left(\mathcal{G}_{\lambda} u, \mathcal{G}_{\lambda} v\right)$.

Let us first consider (1). If $v$ is not in the image of $\mathcal{U}^{-}$then by [Web15, 5.12], we have that $v$ is a summand of $\varepsilon_{i} v^{\prime}$ for some 1-morphism $\mu+\alpha_{i} \rightarrow v$; let $e: \varepsilon_{i} v^{\prime} \rightarrow \varepsilon_{i} v^{\prime}$ by an idempotent whose image is $v$, and $v^{\prime \prime}$ be the image of $1-e$, that is the complementary summand. By assumption, we have a surjection

$$
\operatorname{Hom}_{\mathcal{U}}\left(u, \mathcal{E}_{i} v^{\prime}\right) \cong \operatorname{Hom}_{\mathcal{U}}\left(\mathcal{F}_{i} u, v^{\prime}\right) \rightarrow \operatorname{Hom}_{\mathcal{Q}_{\lambda}}\left(\mathcal{G}_{\lambda}\left(\mathcal{F}_{i} u\right), \mathcal{G}_{\lambda}\left(v^{\prime}\right)\right) \cong \operatorname{Hom}_{\Omega_{\lambda}}\left(\mathcal{G}_{\lambda}(u), \mathcal{G}_{\lambda}\left(\mathcal{E}_{i} v^{\prime}\right)\right) \text {. }
$$

With we compose this map with the idempotent $\mathcal{G}_{\lambda} e$, then we obtain a surjection $\operatorname{Hom}_{\mathcal{U}}\left(u, \mathcal{E}_{i} v^{\prime}\right) \rightarrow \operatorname{Hom}_{2_{\lambda}}\left(\mathcal{G}_{\lambda}(u), \mathcal{G}_{\lambda}(v)\right)$, which kills $\operatorname{Hom}_{\mathcal{U}}\left(u, v^{\prime \prime}\right)$; thus, the induced $\operatorname{map} \operatorname{Hom}_{\mathcal{U}}(u, v) \rightarrow \operatorname{Hom}_{Q_{\lambda}}\left(\mathcal{G}_{\lambda}(u), \mathcal{G}_{\lambda}(v)\right)$ is surjective as desired. Claim (2) follows by a symmetric argument.

Thus, it remains to establish $\operatorname{Hom}_{\mathcal{U}}(u, v) \rightarrow \operatorname{Hom}_{Q_{\lambda}}\left(\mathcal{G}_{\lambda} u, \mathcal{G}_{\lambda} v\right)$ for $u$ in the image of $\mathcal{U}^{-}$and $v$ in the image of $\mathcal{U}^{+}$. For reasons of weight, the target can only be non-zero if $\mu=v$ and $u=v=\mathbb{1}_{\mu}$. Thus, we must prove that

$$
\operatorname{Hom}_{\mathcal{U}}\left(\mathbb{1}_{\mu}, \mathbb{1}_{\mu}\right) \rightarrow \operatorname{Hom}_{\mathbb{Q}_{\lambda}}\left(\mathbb{1}_{\mu}, \mathbb{1}_{\mu}\right) \cong H^{*}\left(\mathfrak{M}_{\mu}^{\lambda}\right) \text {. }
$$

This surjectivity is a consequence of the "algebraic Kirwan surjectivity" discussed in [Weba]. Combining the surjectivity [Weba, ??] to the center of the cyclotomic quotient and the isomorphism [Weba, ??] of said center to the cohomology of the quiver variety, the map of (3.3) must be surjective.

By a standard argument (see, for example, [Webb, Lemma ??]), this shows that the functor $\mathcal{G}_{\lambda}$ sends each indecomposable 1-morphism to an indecomposable bimodule. In fact, we can strengthen this statement: 
Lemma 3.10 For every simple $\mathcal{D}_{X_{\xi}^{\lambda}} \otimes \mathcal{D}_{X_{\xi^{\prime}}^{\lambda}}^{o p}$-module $L$, the reduction $\mathfrak{r}(L)$ is simple, and every simple object in the heart of $\mathcal{Q}$ is a reduction of such a simple module.

Proof. The functor $r$ is exact; thus for any simple $K$ in 2 , all but one composition factor of $\mathfrak{r}_{!}(K)$ must be killed by $r$. Thus, $K$ is the reduction of that composition factor $L$. On the other hand, if $L$ is simple and $\mathfrak{r}(L) \neq 0$, then we have a non-zero map $K \rightarrow \mathfrak{r}(L)$ for some $K$, and thus a map $\mathfrak{r}_{!}(K) \rightarrow L$. Thus, $L$ must be the unique composition factor of $\mathfrak{r}_{!}(K)$ not killed by $\mathfrak{r}$, and so $\mathfrak{r}(L)=K$ and is thus simple.

Fix a vertex $i$ (which we assume to be a source). It will frequently be useful be useful to consider a variety intermediate to imposing all stability conditions and none of them:

Definition 3.11 We let $\hat{X}_{\xi}^{\lambda}$ be the open locus in $X_{\xi}^{\lambda}$ where the sum

$$
x_{\text {out }}: V_{i} \rightarrow W_{i} \oplus \bigoplus_{\alpha(e)=i} V_{\omega(e)}
$$

of the maps along edges pointing out from $i$ is injective. We let $\hat{X}_{\xi \pm \alpha_{i} ; \alpha_{i}}^{\lambda}$ be the restriction of the correspondence $X_{\xi \pm \alpha_{i} ; \alpha_{i}}^{\lambda}$ to the same locus.

Lemma 3.12 The object $\mathcal{G}_{\lambda}(P)$ for $P$ a 1-morphism in $\mathcal{U}$ is isomorphic to $\mathfrak{r}_{1}(M)$ for $M$ a sum of shifts of simple regular holonomic D-modules and is thus a sum of shifts of simple $\mathcal{A}$-modules. If $\tilde{\psi}(P)=P$, then the $D$-module $M$ can be taken to be self-dual.

We should note that this is an analogue of Conjecture 4.13 in [Lia] in our situation.

Proof. In order to show both of these statements, we need only show that for any sequence (i) (including both positive and negative simple roots), the complex $\mathcal{G}_{\lambda}(\mathbf{i})$ is the reduction of a self-dual sum of shifts of regular holonomic D-modules. We induct on the length of $\mathbf{i}$.

First, we note that the identity 1-morphism, the sheaf $\mathcal{A}_{\xi}$ with the diagonal bimodule structure, is simple since it has irreducible support and the diagonal bimodule over the Weyl algebra is simple. It is the analogue of this point which is actually quite difficult in Li's category, and thus is an obstruction to using the techniques described here in that situation. 
We have the diagram of maps

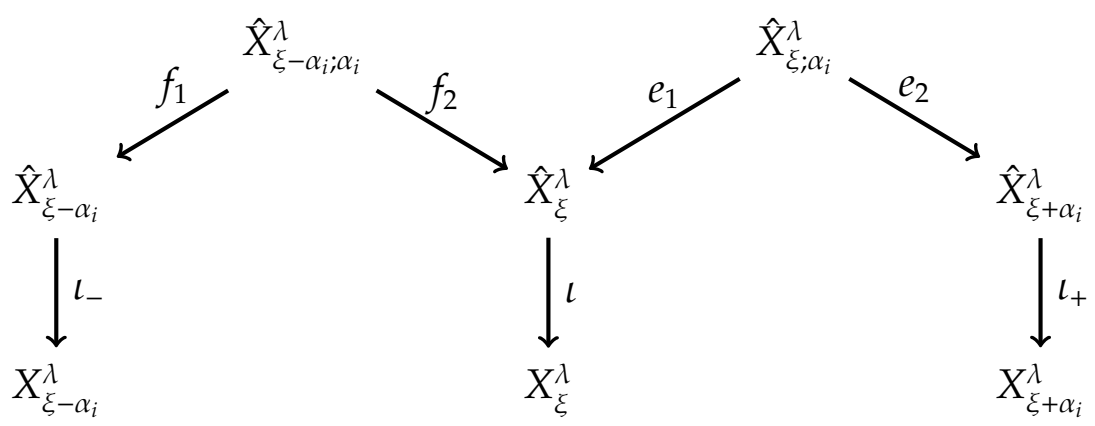

We note that each one of these maps is smooth or an open inclusion. We let

$$
f_{1}^{! *}:=f_{1}^{*}\left[\operatorname{dim} \hat{X}_{\xi-\alpha_{i} ; \alpha_{i}}^{\lambda}-\operatorname{dim} X_{\xi-\alpha_{i}}^{\lambda}\right]=f_{1}^{!}\left[\operatorname{dim} X_{\xi-\alpha_{i}}^{\lambda}-\operatorname{dim} \hat{X}_{\xi-\alpha_{i} ; \alpha_{i}}^{\lambda}\right]
$$

denote the unique shift of the pullback functor with commutes with Verdier duality, and similarly for $f_{2}^{! *}, e_{1}^{! *}, e_{2}^{l *}$. We can consider a simple D-module $L$ on $X_{\xi}^{\lambda}$. Rewriting convolution with $\widehat{\mathscr{F}}_{i}, \hat{\mathscr{E}}_{i}$ in terms of the diagram (3.4), we have that

$$
\mathfrak{r}\left(L \star \hat{\mathscr{E}}_{i}\right)=\mathfrak{r}\left(\left(\iota_{+}\right)_{*}\left(e_{2}\right)_{*} e_{1}^{l * *} \iota^{*} L\right) \quad \mathfrak{r}\left(L \star \hat{\mathscr{F}}_{i}\right)=\mathfrak{r}\left(\left(\iota_{-}\right)_{*}\left(f_{1}\right)_{*} f_{2}^{l *} \iota^{*} L\right) .
$$

Furthermore, applying Lemma 4.5, we have that

$$
\mathfrak{r}(L) \star \mathscr{E}_{i}=\mathfrak{r}\left(\left(\iota_{+}\right)_{*}\left(e_{2}\right)_{*} e_{1}^{l * *} \iota^{*} L\right) \quad \mathfrak{r}(L) \star \mathscr{F}_{i}=\mathfrak{r}\left(\left(\iota_{-}\right)_{*}\left(f_{1}\right)_{*} f_{2}^{\prime *} \iota^{*} L\right) .
$$

By the Decomposition Theorem, the D-modules $\left(e_{2}\right)_{*} e_{1}^{* *} \iota^{*} L$ and $\left(f_{1}\right)_{*} f_{2}^{! * *} l^{*} L$ are a sum of shifts of simple D-modules on $\hat{X}_{\xi \pm \alpha_{i}}^{\lambda}$; furthermore, since all D-modules supported on $X_{\xi \pm \alpha_{i}}^{\lambda} \backslash \hat{X}_{\xi \pm \alpha_{i}}^{\lambda}$ are killed by $\mathrm{r}$, we can replace $\left(\iota_{ \pm}\right)_{*}$ with the intermediate extension $\left(\iota_{ \pm}\right)_{: *}$ in (3.5):

$$
\mathfrak{r}(L) \star \mathscr{E}_{i}=\mathfrak{r}\left(\left(L_{+}\right)_{! *}\left(e_{2}\right)_{*} e_{1}^{l *} \iota^{*} L\right) \quad \mathfrak{r}(L) \star \mathscr{F}_{i}=\mathfrak{r}\left(\left(L_{-}\right)_{* !}\left(f_{1}\right)_{*} f_{2}^{f *} \iota^{*} L\right)
$$

Since intermediate extension preserves simplicity, we see that $\mathrm{r}(L) \star \mathscr{E}_{i}$ is a reduction of sum of shifts of simple $\mathcal{A}_{\xi \pm \alpha_{i}}$-modules by Lemma 3.10. By the inductive assumption, $\mathcal{G}_{\lambda}(\mathbf{i})=\mathrm{r}(M)$ for $M$ a self-dual sum of shifts of regular holonomic D-modules. Thus $\mathcal{G}_{\lambda}(\mathbf{i}, \pm i)$ is also a reduction of a self-dual sum of shifts of regular holonomic D-modules (either $\left(\iota_{-}\right)_{* !}\left(f_{1}\right)_{*} f_{2}^{! *} \iota^{*} M$ or $\left.\left(l_{+}\right)_{! *}\left(e_{2}\right)_{*} e_{1}^{! *} l^{*} M\right)$ since the operations $\left(\iota_{-}\right)_{* !}, l^{*},\left(f_{1}\right)_{*}, f_{2}^{! *},\left(e_{2}\right)_{*}$ and $e_{1}^{l *}$ all commute with duality (since $f_{i}, e_{i}$ are proper and smooth).

Combining Lemma 3.12 with the observation that indecomposability is preserved under this map, we see that:

Corollary 3.13 For an indecomposable 1-morphism $P$ in $\mathcal{U}$, the sheaf $\mathcal{G}_{\lambda}(P)$ is simple.

Let $Q_{\lambda}$ denote the image of $\mathcal{G}_{\lambda}$ as a functor between graded additive categories, where the grading on the former arises from the homological grading; this is a full 2subcategory of $Q_{\lambda}$, which is closed under convolution (but not under extensions). This is a mixed humorous category in the sense of [Web15, 1.11] by applying [Web15, 1.20] with $\mathcal{J}$ given by the dg-subcategory $Q_{\lambda}$ generates equipped with the usual $t$-structure. 
Thus, if we let the canonical basis of $K_{q}\left(Q_{\lambda}\right)$ be the classes of the simple modules, then [Web15, 1.15] implies that these are also canonical bases in the algebraic sense of bar-invariant almost-orthogonal vectors.

Convolution also endows the graded Grothendieck group $K_{q}\left(Q_{\lambda}\right)$ with an algebra, with an induced algebra map $K_{q}\left(\mathcal{G}_{\lambda}\right): K_{q}(\mathcal{U}) \rightarrow K_{q}\left(Q_{\lambda}\right)$. Finally, [Web15, 1.17] shows that:

Proposition 3.14 Each canonical basis vector in $K_{q}\left(Q_{\lambda}\right)$ is the image of a unique canonical basis vector in $\dot{\mathbf{U}}_{q} \cong K_{q}(\mathcal{U})$, and any other canonical basis vector in $\dot{\mathbf{U}}_{q} \cong K_{q}(\mathcal{U})$ in killed by $K_{q}\left(G_{\lambda}\right)$.

3.4. Decategorification. Finally, we turn to understanding how this action decategorifies. As defined in [BPW, §6.2], based on work of Kashiwara and Schapira [KS], we have a map $C C$ from the K-group of sheaves supported on $Z$ to $H_{\text {top }}^{B M}(Z)$ which intertwines convolution of sheaves with convolution of Borel-Moore classes. Composing the map induced on Grothendieck groups defined by $\mathcal{G}_{\lambda}$ with $C C$, we obtain a homomorphism $C: K(\mathcal{U}) \rightarrow H_{\text {top }}^{B M}(Z)$.

Proposition 3.15 We have a commutative diagram

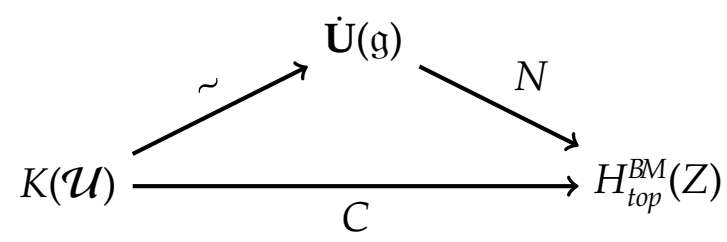

where $N: \dot{\mathbf{U}}(\mathfrak{g}) \rightarrow H_{\text {top }}^{B M}(Z)$ is the map defined by Nakajima in [Nak98].

Proof. We can fix a vertex $i$, and assume that we have chosen our orientation so that $i$ is a source. Let $\hat{X}_{\xi}^{\lambda}$ and $\hat{X}_{\xi \pm \alpha_{i} ; \alpha_{i}}^{\lambda}$ be as defined in 3.11. We can define a bimodule $\hat{\mathscr{E}}_{i}$ restricting $\tilde{\mathscr{E}}_{i}$ to the hatted varieties. We still have a reduction functor $\hat{\mathfrak{x}}$ on D-modules over $\hat{X}_{\xi}^{\lambda}$, since any point where $x_{\text {out }}$ is not injective is destabilized by a subrepresentation on $i$ given by its kernel, and $\hat{\mathfrak{r}}\left(\hat{\mathscr{E}}_{i}\right)=\mathscr{E}_{i}$.

The map CC sends $\left[\mathscr{E}_{i}\right]$ to the sum of the fundamental classes of the components of its support variety, weighted by the generic dimension of the stalk of its classical limit at a generic point of the component. However, the map $\hat{X}_{\xi ; \alpha_{i}}^{\lambda} \rightarrow \hat{X}_{\xi}^{\lambda} \times \hat{X}_{\xi-\alpha_{i}}^{\lambda}$ is injective with smooth image; it is locally modeled on the map from the $(k, k+1)$-type partial flag variety to the 2 Grassmannians (with the ambient space given by the sum $\left.W_{i} \oplus \bigoplus_{\alpha(e)=i} V_{\omega(e)}\right)$. Thus, its pushforward has irreducible characteristic variety with multiplicity one.

The intersection of this characteristic variety with the stable locus is the support variety of $\mathscr{E}_{i}$, which we can thus identify with the Hecke correspondence denoted $\mathfrak{P}_{i}$ in [Nak98]. By a symmetric argument, the support variety of $\mathscr{F}_{i}$ is the variety obtained 
from this one by reversing factors. Thus, we have that

$$
\left[\mathcal{E}_{i}\right] \mapsto\left[\mathscr{E}_{i}\right] \mapsto\left[\mathfrak{P}_{i}\right] \quad\left[\mathcal{F}_{i}\right] \mapsto\left[\mathscr{F}_{i}\right] \mapsto\left[\omega\left(\mathfrak{P}_{i}\right)\right]
$$

By [Nak98, 9.4], the homomorphism $N: \dot{U} \rightarrow H_{\text {top }}^{B M}(\mathrm{Z})$ is the unique one with this property.

Similarly, in [KS, 6.5.4], it's shown than the action of $K(\mathcal{U})$ on $K\left(C^{\lambda}\right)$ by convolution is intertwined by $C C$ with the Nakajima's action of $U(\mathfrak{g})$ on top Borel-Moore homology of the core $L^{\lambda}:=\sqcup_{\xi} L_{\xi}^{\lambda}$. That is:

Corollary 3.16 The diagram (3.6) can be extended to a commutative diagram including the natural actions of $K(\mathcal{U})$ on $K\left(C^{\lambda}\right)$ induced by $\mathcal{G}_{\lambda}$, Nakajima's action of $H_{\text {top }}^{B M}(Z)$ on $H_{\text {top }}^{B M}\left(L^{\lambda}\right)$, and the usual action of $U(\mathfrak{g})$ on $V_{\lambda}$ :

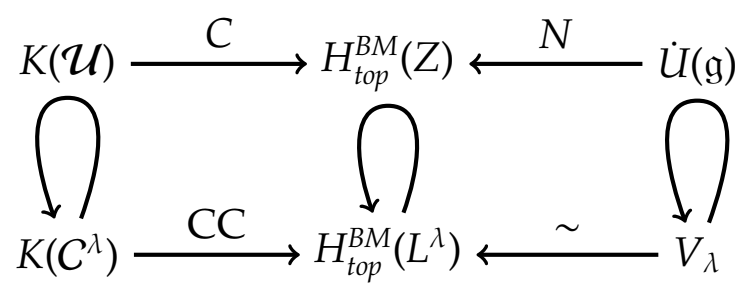

\section{The proof of Theorem 3.1}

Now we proceed to the proof of Theorem 3.1 through a series of lemmata. Let $m_{\mu}^{\lambda}=\operatorname{dim} \mathfrak{M}_{\mu}^{\lambda}$

Lemma 4.1 The left and right adjoint of $\mathscr{F}_{i} \star-$ are the convolution functors

$$
\mathscr{E}_{i}\left[1 / 2\left(m_{\mu}^{\lambda}-m_{\mu-\alpha_{i}}^{\lambda}\right)\right] \star-\quad \text { and } \quad \mathscr{E}_{i}\left[1 / 2\left(m_{\mu-\alpha_{i}}^{\lambda}-m_{\mu}^{\lambda}\right)\right] \star-.
$$

Proof. As in [KS, (2.3.18)], we can take the dual

$$
\mathbb{D} \mathscr{E}_{i} \cong \mathbb{R} \mathcal{H} o m_{\mathcal{A}_{\mu} \otimes \mathcal{A}_{\mu-\alpha_{i}}^{o p}}\left(\mathscr{E}_{i}, \mathcal{A}_{\mu} \otimes \mathcal{A}_{\mu-\alpha_{i}}^{o p}\right)
$$

This a left $\mathcal{A}_{\mu-\alpha_{i}}$-module and right $\mathcal{A}_{\mu}$-module By [KS, 2.3.15], its is the shift of a $\mathcal{A}_{\mu-\alpha_{i}} \otimes \mathcal{A}_{\mu}$ module with Lagrangian support equal to $\left.\omega\left(\mathfrak{P}_{i}\right)\right]$. Thus, we must have $\mathbb{D} \mathscr{E}_{i}\left[-1 / 2\left(m_{\mu}^{\lambda}+m_{\mu-\alpha_{i}}^{\lambda}\right)\right] \cong \mathscr{F}_{i}$; this is also easily shown using local computations with D-modules. By [KS, 6.2.4], this homological shift is just convolution with the square root of the dualizing sheaf for $\mathcal{A}_{\mu-\alpha_{i}} \otimes \mathcal{A}_{\mu}$. We denote the dualizing sheaf for $\mathcal{A}_{\mu}$ by $\omega_{\mu}$. Similarly, $\mathbb{D} \mathscr{F}_{i}\left[-1 / 2\left(m_{\mu}^{\lambda}+m_{\mu-\alpha_{i}}^{\lambda}\right) \cong \mathscr{E}_{i}\right.$. For any $\mathcal{A}_{\mu}$-module $\mathcal{M}$ and $\mathcal{A}_{\mu-\alpha_{i}}$-module $\mathcal{N}$, 
we have that

$$
\begin{aligned}
\mathbb{R H o m}_{\mathcal{A}_{\mu}}\left(\mathcal{M}, \mathscr{F}_{i} \star \mathcal{N}\right) & \cong \mathbb{D} \mathcal{M} \star\left(\mathscr{F}_{i} \star \mathcal{N}\right) \\
& \cong\left(\mathbb{D} \mathcal{M} \star \mathscr{F}_{i}\right) \star \mathcal{N} \\
& \cong\left(\mathbb{D} \mathcal{M} \star \omega_{\mu-\alpha_{i}}^{1 / 2} \star \mathbb{D} \mathscr{E}_{i} \star \omega_{\mu}^{1 / 2}\right) \star \mathcal{N} \\
& \cong \mathbb{D}\left(\mathscr{E}_{i} \star \mathcal{M}\right) \star \mathcal{N}\left[1 / 2\left(m_{\mu-\alpha_{i}}^{\lambda}-m_{\mu}^{\lambda}\right)\right] \\
& \cong \mathbb{R} \mathcal{H} o m_{\mathcal{A}_{\mu}}\left(\mathscr{E}_{i} \star \mathcal{M}\left[1 / 2\left(m_{\mu}^{\lambda}-m_{\mu-\alpha_{i}}^{\lambda}\right], \mathcal{N}\right) .\right.
\end{aligned}
$$

By symmetry, this gives the biadjunction.

Lemma 4.2 We have a natural isomorphism $M \star \mathfrak{r} N \cong \mathfrak{r}\left(\mathfrak{r}_{*} M \star N\right)$ for any complex of $\mathcal{A}_{\xi} \otimes \mathcal{A}_{\xi^{\prime}}^{o p}$-modules $M$ and any complex of $\mathcal{D}_{X_{\xi^{\prime}}^{\lambda}} \otimes \mathcal{D}_{X_{\xi^{\prime \prime}}^{\lambda}}^{o p}$-modules $N$.

Proof. We need only confirm this isomorphism when $M=M_{1} \otimes M_{2}$ and $N=\mathcal{D}_{X_{\xi^{\prime}}^{\lambda}} \otimes \mathcal{D}_{X_{\xi^{\prime \prime}}^{\lambda}}$. In this case,

$$
M \star r N \cong M_{1} \otimes \Gamma\left(\mathfrak{M}_{\xi^{\prime}}^{\lambda} ; M_{2}\right) \otimes \mathcal{A}_{\xi^{\prime \prime}} \quad \mathfrak{r}\left(\mathfrak{r}_{*} M \star N\right) \cong M_{1} \otimes \Gamma\left(X_{\xi^{\prime}}^{\lambda} ; \mathfrak{r}_{*} M_{2}\right) \otimes \mathcal{A}_{\xi^{\prime \prime}}
$$

The result then follows from the isomorphism

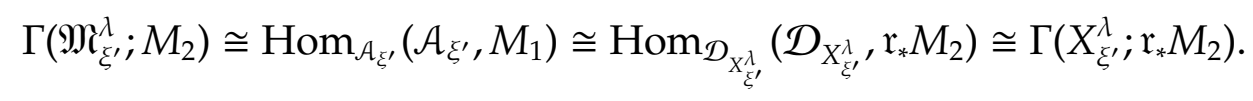

Lemma 4.3 If $\mathcal{M}$ is a $\mathcal{D}_{X_{\varepsilon}^{\lambda}}$-module whose microsupport $\mu \operatorname{supp}(\mathcal{M})$ is contained in the unstable locus, then $\mu \operatorname{supp}\left(\mathcal{M} \star \tilde{\mathscr{E}}_{i}\right)$ is also contained in the unstable locus. That is, if $\mathrm{r}(\mathcal{M})=0$, then $\mathrm{r}\left(\mathcal{M} \star \tilde{\mathscr{E}}_{i}\right)=0$.

Proof. Since the map $p_{1} \times p_{2}$ is a composition of a closed inclusion and a smooth map, we can apply the description of the effect of these maps on singular supports given in [Ber, 9a \& b]. Thus, the microsupport $\mu \operatorname{supp}\left(\tilde{\mathscr{E}}_{i}\right)$ lies in the image in $T^{*} X_{\xi}^{\lambda} \times T^{*} X_{\xi-\alpha_{i}}^{\lambda}$ of

$$
\left\{x \in X_{\xi ; \alpha_{i}}^{\lambda} \varphi \in T_{\left(p_{1}(x), p_{2}(x)\right)}^{*}\left(X_{\xi}^{\lambda} \times X_{\xi-\alpha_{i}}^{\lambda}\right) \mid\left(p_{1} \times p_{2}\right)^{*} \varphi=0\right\} .
$$

We can think of $x$ as a representation of the oriented quiver with chosen subrepresentation, the covector $\varphi$ as a choice of maps along the oppositely oriented arrows that extend the total representation and the subrepresentation to representations of the preprojective algebra; in this case, the vanishing condition is simply that the inclusion is a map of preprojective representations. That is, the microsupport of $\tilde{\mathscr{O}}_{i}$ is composed of the pairs of representations of the preprojective algebra such that the LHS is isomorphic to a subrepresentation of the RHS. In particular, if the lefthand point $p_{1}(x)$ has a destabilizing subrepresentation, $p_{2}(x)$ does as well. That is, the microsupport of $\tilde{\mathscr{E}}_{i}$ has the property that if $p_{1}(x)$ is unstable, then $p_{2}(x)$ is as well.

Applying the same result from [Ber, $9 \mathrm{a} \& \mathrm{~b}]$, the microsupport $\mu \operatorname{supp}\left(\mathcal{M} \star \tilde{\mathscr{E}}_{i}\right)$ is contained in the set

$$
\left\{(x, \varphi) \in T^{*} X_{\xi-\alpha_{i}}^{\lambda} \mid\left(x^{\prime}, \varphi^{\prime} ; x, \varphi\right) \in \mu \operatorname{supp}\left(\tilde{\mathscr{E}}_{i}\right) \text { for some }\left(x^{\prime}, \varphi^{\prime}\right) \in \mu \operatorname{supp}(\mathcal{M}) .\right\}
$$


This shows that all points in this microsupport must be unstable.

Lemma $4.4 \mathfrak{r}\left(\tilde{\mathscr{E}}_{i_{1}} \star \cdots \star \tilde{\mathscr{E}}_{i_{n}}\right) \cong \mathscr{E}_{i_{1}} \star \cdots \star \mathscr{E}_{i_{n}}$

Proof. We induct on $n$; when $n=1$, this is true by definition.

By the inductive hypothesis, $\mathfrak{r}\left(\tilde{\mathscr{E}}_{i_{1}} \star \cdots \star \tilde{\mathscr{E}}_{i_{n-1}}\right) \cong \mathscr{E}_{i_{1}} \star \cdots \star \mathscr{E}_{i_{n-1}}$. Thus, we have maps

$$
\mathfrak{r}_{1}\left(\mathscr{E}_{i_{1}} \star \cdots \star \mathscr{E}_{i_{n-1}}\right) \rightarrow \tilde{\mathscr{E}}_{i_{1}} \star \cdots \star \tilde{\mathscr{E}}_{i_{n-1}} \rightarrow \mathfrak{r}_{*}\left(\mathscr{E}_{i_{1}} \star \cdots \star \mathscr{E}_{i_{n-1}}\right)
$$

which induce isomorphisms after applying $\mathrm{r}$.

We have a map $a: \mathfrak{r}_{!}\left(\mathscr{E}_{i_{1}} \star \cdots \star \mathscr{E}_{i_{n-1}}\right) \rightarrow \tilde{\mathscr{E}}_{i_{1}} \star \cdots \star \tilde{\mathscr{E}}_{i_{n-1}}$ which induces an isomorphism after applying $\mathrm{r}$. Thus $C(a)$, the cone of this morphism, has cohomology microsupported on the unstable locus. By definition, we have an exact triangle

$$
r_{!}\left(\mathscr{E}_{i_{1}} \star \cdots \star \mathscr{E}_{i_{n-1}}\right) \rightarrow \tilde{\tilde{E}_{i_{1}}} \star \cdots \star \tilde{\mathscr{E}}_{i_{n-1}} \rightarrow C(a) \stackrel{[1]}{\rightarrow}
$$

Thus, applying the triangulated functor $-\star \widetilde{\mathscr{E}}_{i_{n}}$ to the equation (4.1), we have an exact triangle

$$
\mathfrak{r}_{!}\left(\mathscr{E}_{i_{1}} \star \cdots \star \mathscr{E}_{i_{n-1}}\right) \star \tilde{\mathscr{E}}_{i_{n}} \rightarrow \tilde{\mathscr{E}}_{i_{1}} \star \cdots \star \tilde{\mathscr{E}}_{i_{n}} \rightarrow C(a) \star \tilde{\mathscr{E}}_{i_{n}} \stackrel{[1]}{\rightarrow} .
$$

By Lemma 4.3, we have $\mathfrak{r}\left(C(a) \star \tilde{\mathscr{E}}_{i_{n}}\right)=0$, so applying $\mathfrak{r}$ to this exact triangle shows that

$$
\mathfrak{r}\left(\mathfrak{r}_{!}\left(\mathscr{E}_{i_{1}} \star \cdots \star \mathscr{E}_{i_{n-1}}\right) \star \tilde{\mathscr{E}}_{i_{n}}\right) \cong \mathfrak{r}\left(\tilde{\mathscr{E}}_{i_{1}} \star \cdots \star \tilde{\mathscr{E}}_{i_{n}}\right)
$$

If we apply Lemma 4.2 with $M=\mathscr{E}_{i_{1}} \star \cdots \star \mathscr{E}_{i_{n-1}}$ and $N=\tilde{\mathscr{E}}_{i_{n}}$, we arrive at

$$
\mathscr{E}_{i_{1}} \star \cdots \star \mathscr{E}_{i_{n}} \cong \mathfrak{r}\left(\mathfrak{r}_{!}\left(\mathscr{E}_{i_{1}} \star \cdots \star \mathscr{E}_{i_{n-1}}\right) \star \tilde{\mathscr{E}}_{i_{n}}\right) \text {. }
$$

Combining equations (4.2-4.3), we arrive at the desired isomorphism

$$
\mathscr{E}_{i_{1}} \star \cdots \star \mathscr{E}_{i_{n}} \cong \mathfrak{r}\left(\mathfrak{r}_{!}\left(\mathscr{E}_{i_{1}} \star \cdots \star \mathscr{E}_{i_{n-1}}\right) \star \tilde{\mathscr{E}}_{i_{n}}\right) \cong \mathfrak{r}\left(\tilde{\mathscr{E}}_{i_{1}} \star \cdots \star \tilde{\mathscr{E}}_{i_{n}}\right) .
$$

Lemma 4.5 For any $\mathcal{D}_{X_{\mu}^{\lambda}}$-module $\mathcal{M}$, we have that:

$$
\mathfrak{r} \mathcal{M} \star \mathscr{F}_{i} \cong \mathfrak{r}\left(\mathcal{M} \star \hat{\mathscr{F}}_{i}\right) \quad \mathfrak{r} \mathcal{M} \star \mathscr{E}_{i} \cong \mathfrak{r}\left(\mathcal{M} \star \hat{\mathscr{E}}_{i}\right)
$$

Proof. We let $\hat{O}_{\xi}^{\lambda}=T^{*} \hat{X}_{\xi}^{\lambda} \backslash \mathfrak{M}_{\xi}^{\lambda}$ and

$$
\hat{I}: \mathfrak{M}_{\xi}^{\lambda} \times \mathfrak{M}_{\xi+\alpha_{i}}^{\lambda} \rightarrow T^{*} X_{\xi}^{\lambda} \times \mathfrak{M}_{\xi-\alpha_{i}}^{\lambda} \quad \hat{J}: O_{\xi}^{\lambda} \times \mathfrak{M}_{\xi+\alpha_{i}}^{\lambda} \rightarrow T^{*} \hat{X}_{\xi}^{\lambda} \times \mathfrak{M}_{\xi-\alpha_{i}}^{\lambda}
$$

be the inclusion of the loci where the the first coordinate is (un)stable. By definition,

$$
\mathfrak{r} \mathcal{M} \star \mathscr{F}_{i} \cong\left(p_{2}\right)_{*} \hat{I}_{*} \hat{I}^{*}\left(p_{1}^{*} \underline{\mu} \mathcal{M} \otimes \underline{\mu} \underline{\mathscr{F}}_{i}\right) \quad \mathfrak{r}\left(\mathcal{M} \star \hat{\mathscr{F}}_{i}\right) \cong\left(p_{2}\right)_{*}\left(p_{1}^{*} \underline{\mu} \mathcal{M} \otimes \underline{\mu}_{\hat{F}_{i}}\right)
$$

Thus, by the usual recollement, these will be isomorphic via the natural map if and only if $\left(p_{2}\right)_{*} \hat{J}_{!} \hat{J}^{!}\left(p_{1}^{*} \boldsymbol{\mu} \mathcal{M} \otimes \boldsymbol{\mu} \hat{\mathscr{F}}_{i}\right)=0$. Thus, it suffices to show that $\hat{\jmath}^{!}\left(p_{1}^{*} \boldsymbol{\mu} \mathcal{M} \otimes \boldsymbol{\mu} \hat{\mathscr{F}}_{i}\right)=0$. Any point which lies in its support must have the following properties: its two coordinates correspond to framed modules $S_{1}, S_{2}$ over the preprojective algebra with an inclusion $S_{2} \hookrightarrow S_{1}$, such that $S_{2}$ is stable, and $S_{1}$ has a destabilizing subrepresentation $Z_{1} \subset S_{1}$. Furthermore, this destabilizing subrepresentation cannot lie solely on the vertex $i$. 
However, the cokernel $S_{1} / S_{2}$ is only supported on $i$, so $Z_{1}$ cannot inject into this quotient. The intersection $Z_{1} \cap S_{2}$ must thus be non-trivial, providing a destabilizing subrepresentation of $S_{2}$. Thus, we have arrived at a contradiction, and this sheaf must have empty support, and thus be 0 . The second equation follows by a similar argument $T^{*} X_{\xi}^{\lambda} \times \mathfrak{M}_{\xi+\alpha_{i}}^{\lambda}$ or alternately, from Lemma 4.3.

Proof of Theorem 3.1. We wish to check the conditions of [Roub, 4.13]. This is defined by a list of conditions, which we check in the same order.

- the functors $\mathscr{E}_{i} \star-$ and $\mathscr{F}_{i} \star-$ are biadjoint up shift. This follows from Lemma 4.1 .

- the sheaves $\oplus_{\mathbf{i}} \mathscr{E}_{i_{1}} \star \cdots \star \mathscr{E}_{i_{n}}$ carry an action of the KLR algebra for the polynomials $Q$ we have specified. The solution sheaf (i.e. the image under the RiemannHilbert correspondence) of $\tilde{\mathscr{E}}_{i_{1}} \star \cdots \star \tilde{\mathscr{E}}_{i_{n}}$ is precisely the perverse sheaf that Varagnolo and Vasserot denote by ${ }^{\delta} \mathcal{L}_{\mathbf{i}}$ in [VV11].

In our language, [VV11, 3.5] and [Roub, 5.7] (independently) show that the Ext algebra of solution sheaves of $\oplus_{\mathbf{i}} \tilde{\mathscr{E}}_{i_{1}} \star \cdots \star \tilde{\mathscr{E}}_{i_{n}}$ is given by the KLR algebra $R=\oplus R_{v}$; since the Riemann-Hilbert correspondence is an equivalence of categories, we arrive at an isomorphism

$$
\operatorname{Ext}^{\bullet}\left(\oplus_{\mathbf{i}} \tilde{\mathscr{E}}_{i_{1}} \star \cdots \star \tilde{\mathscr{E}}_{i_{n}}\right) \cong R
$$

It follows that the image of these sheaves under any functor, in particular $\mathfrak{r}\left(\tilde{\mathscr{E}}_{i_{1}} \star \cdots \star \tilde{\mathscr{E}}_{i_{n}}\right) \cong \mathscr{E}_{i_{1}} \star \cdots \star \mathscr{E}_{i_{n}}$, still carry this action.

- The functors $\mathscr{E}_{i} \star-$ and $\mathscr{F}_{i} \star-$ are locally nilpotent. This follows from that fact that for fixed $\xi$, there are only finitely many integers such that $\mathfrak{M}_{\xi+k \alpha_{i}}^{\lambda}$ is non-empty.

The final condition is that for each $i$, we have

$$
\begin{aligned}
& \mathscr{F}_{i} \star \mathscr{E}_{i} \cong \mathscr{E}_{j} \star \mathscr{F}_{i} \oplus\left(q^{\left\langle\alpha_{i}, \xi\right\rangle+d_{i}}+\cdots+q^{-\left\langle\alpha_{i}, \xi\right\rangle-d_{i}}\right) \cdot \mathcal{A}_{\xi} \quad \text { if }\left\langle\alpha_{i}, \xi\right\rangle \leq 0 \\
& \mathscr{F}_{i} \star \mathscr{E}_{i} \oplus\left(q^{-\left\langle\alpha_{i}, \xi\right\rangle+d_{i}}+\cdots+q^{\left\langle\alpha_{i}, \xi\right\rangle-d_{i}}\right) \cdot \mathcal{A}_{\xi} \cong \mathscr{E}_{j} \star \mathscr{F}_{i} \quad \text { if }\left\langle\alpha_{i}, \xi\right\rangle \geq 0 .
\end{aligned}
$$

Once this is proven, the result will follow. This can be proven from calculations done on the level of constructible sheaves carried through the Riemann-Hilbert correspondence. That these isomorphisms exist is shown in the characteristic $p$ setting by [Lib, 1.12-13] following similar proofs of Zheng [Zhe]. Li's proofs use no special facts about characteristic $p$ fields; in principle, we could simply cite his work, but for the sake of completeness, we give arguments in the deformation quantization setting for the same facts.

Applying Fourier transform as necessary, we can assume that $i$ is a source. Proposition 3.2 assures us that we can transition between the different quantizations that arise from different orientations; the categories of modules over the different quantizations that arise are given by tensor product with quantizations of line bundles to bimodules given in [BPW, 5.2]. It's easily seen that these intertwine the actions of $\mathcal{U}$. 
We consider the diagram (3.4) of maps again. Recall that the maps $e_{i}$ and $f_{i}$ are both proper and smooth; their fibers are projective spaces. We let $\hat{\mathscr{F}}_{i}$ denote the restriction of $\widetilde{\mathscr{F}}_{i}$ to the injective locus, and similarly for $\hat{\mathscr{E}}_{i}$.

We have an isomorphism of $\hat{\mathscr{E}}_{i} \star \hat{\mathscr{F}}_{i}$ with the pushforward by $\mathbb{f}_{2}:=f_{2} \times f_{2}$ of the structure sheaf of $\hat{X}_{\xi ; \alpha_{i}}^{\lambda} \times_{\hat{X}_{\xi-\alpha_{i}}^{\lambda}} \hat{X}_{\xi ; \alpha_{i}}^{\lambda}$ tensored with the canonical sheaf of the right factor. The sheaf $\hat{\mathscr{F}}_{i} \star \hat{\mathscr{E}}_{i}$ is derived in the same way from $\mathbb{e}_{1}:=e_{1} \times e_{1}$.

Now, we turn to showing that

$$
\begin{aligned}
& \hat{\mathscr{F}}_{i} \star \hat{\mathscr{E}}_{i} \cong \hat{\mathscr{E}}_{j} \star \hat{\mathscr{F}}_{i} \oplus\left(q^{\left\langle\alpha_{i}, \xi\right\rangle+1}+\cdots+q^{-\left\langle\alpha_{i}, \xi\right\rangle-1}\right) \cdot \mathcal{D}_{\Delta} \quad \text { if }\left\langle\alpha_{i}, \xi\right\rangle \leq 0 \\
& \hat{\mathscr{F}}_{i} \star \hat{\mathscr{E}}_{i} \oplus\left(q^{-\left\langle\alpha_{i}, \xi\right\rangle+1}+\cdots+q^{\left\langle\alpha_{i}, \xi\right\rangle-1}\right) \cdot \mathcal{D}_{\Delta} \cong \hat{\mathscr{E}}_{j} \star \hat{\mathscr{F}}_{i} \quad \text { if }\left\langle\alpha_{i}, \xi\right\rangle \geq 0
\end{aligned}
$$

where $\Delta$ denotes the diagonal in $\hat{X}_{\xi}^{\lambda} \times \hat{X}_{\xi}^{\lambda}$. The analogous calculation for $\ell$-adic sheaves is done by $\mathrm{Li}$ in [Lib, 1.13]; specifically, his equations [Lib, (19-20)] compute the two sides of the proceeding displayed equations and show that they agree.

Since his proof is not especially difficult, let us give an account for the reader. The maps

$$
\mathbb{f}_{2}: \hat{X}_{\xi ; \alpha_{i}}^{\lambda} \times_{\hat{X}_{\xi-\alpha_{i}}^{\lambda}} \hat{X}_{\xi ; \alpha_{i}}^{\lambda} \rightarrow \hat{X}_{\xi}^{\lambda} \times \hat{X}_{\xi}^{\lambda} \quad \mathbb{E}_{1}: \hat{X}_{\xi+\alpha_{i} ; \alpha_{i}}^{\lambda} \times_{\hat{X}_{\xi \xi+\alpha_{i}}^{\lambda}} \hat{X}_{\xi+\alpha_{i} ; \alpha_{i}}^{\lambda} \rightarrow \hat{X}_{\xi}^{\lambda} \times \hat{X}_{\xi}^{\lambda}
$$

both have image given by the set $H_{\xi}^{\lambda}$ of representations (injective at $i$ ) which are the same away from $i$, and where the subspaces at $i$ have intersection of codimension 1 in both spaces. Both $\mathbb{f}_{2}$ and $\mathbb{e}_{1}$ induce an isomorphism on the locus in $H_{\xi}^{\lambda}$ where the representations differ, and a projective space bundle at the points where they coincide.

The map $\mathbb{e}_{1}$ has fiber over the diagonal given by $\mathbb{P}^{v_{i}-1}$, since the fiber consists of all the ways of choosing a hyperplane in $V_{i}$. The map $\mathbb{f}_{2}$ has fiber $\mathbb{P}^{\left\langle\alpha_{i}, \xi\right\rangle+v_{i}-1}$, since the fiber consists of the lines in the cokernel of $x_{\text {out }}$. Finally, the diagonal has codimension $\left\langle\alpha_{i}, \xi\right\rangle+2 v_{i}-1$ inside $H_{\xi}^{\lambda}$. Thus, the map $\mathbb{e}_{1}$ is small if $\left\langle\alpha_{i}, \xi\right\rangle \geq 0$, and the map $\mathbb{t}_{2}$ is small if $\left\langle\alpha_{i}, \xi\right\rangle \leq 0$. Let's reduce to the former case for simplicity.

In this case, $\hat{\mathscr{F}}_{i} \star \hat{\mathscr{E}}_{i}$ is an irreducible D-module $Q$, the unique one on $H_{\xi}^{\lambda}$ which extends the pullback of the canonical sheaf by the first projection, since it is the pushforward by a small resolution of singularities. On the other hand, $\hat{\mathscr{E}}_{i} \star \hat{\mathscr{F}}_{i}$ is the pushforward of a resolution of singularities which is not necessarily small, and is thus of the form $Q \oplus Q^{\prime}$ where $Q^{\prime}$ is a sum of shifts of semi-simple D-modules supported on the diagonal.

Note that $\hat{X}_{\xi}^{\lambda}$ is the quotient of an affine bundle over a Grassmannian by a connected algebraic group, and thus simply connected. Therefore the pullback of $\hat{\mathscr{E}}_{i} \star \hat{\mathscr{F}}_{i}$ to the diagonal is the pushforward by a proper algebraic fiber bundle to a simply connected space, and thus a sum of shifts of the structure sheaf. When we use Kashiwara's theorem to think of this as a D-module on $\hat{X}_{\xi}^{\lambda} \times \hat{X}_{\xi}^{\lambda}$, we obtain a sum of shifts of $\mathcal{D}_{\Delta}$. Furthermore, since the fiber is $\mathbb{P}^{\left\langle\alpha_{i}, \xi\right\rangle+v_{i}-1}$, we know that it is the sum $\left(q^{\left\langle\alpha_{i}, \xi\right\rangle+v_{i}-1}+\cdots+\right.$ $\left.q^{-\left\langle\alpha_{i}, \xi\right\rangle+1}\right) \cdot \mathcal{D}_{\Delta}$. On the other hand, the pullback of $Q$ is $\left(q^{\left\langle\alpha_{i}, \xi\right\rangle+v_{i}-1}+\cdots+q^{\left\langle\alpha_{i}, \xi\right\rangle+1}\right) \cdot \mathcal{D}_{\Delta}$ by 
the same argument. This is only possible if

$$
Q^{\prime} \cong\left(q^{-\left\langle\alpha_{i}, \xi\right\rangle+1}+\cdots+q^{\left\langle\alpha_{i}, \xi\right\rangle-1}\right) \cdot \mathcal{D}_{\Delta} .
$$

This shows that (4.5b) holds. If we instead $\left\langle\alpha_{i}, \xi\right\rangle \leq 0$, we can show (4.5a) by applying the same argument, switching the roles of the two sheaves.

Thus, applying (4.4) to the equations (4.5a 4.5b), we arrive at the desired isomorphisms (4.4a-4.4b). Thus, by [Roub, 4.13], we have a 2 -functor from $\mathcal{U}$ to $Q^{\lambda}$ as desired.

This completes the proof of Theorem A,

\section{ReFERENCES}

[BB81] Alexander Beilinson and Joseph Bernstein, Localisation de g-modules, C. R. Acad. Sci. Paris Sér. I Math. 292 (1981), no. 1, 15-18. MR MR610137 (82k:14015)

[Ber] Joseph Bernstein, Algebraic theory of D-modules, preprint.

[BG] Vladimir Baranovsky and Victor Ginzburg, personal communication.

[BK04] Roman Bezrukavnikov and Dmitry Kaledin, Fedosov quantization in algebraic context, Mosc. Math. J. 4 (2004), no. 3, 559-592, 782. MR MR2119140 (2006j:53130)

[BL] R. Bezrukavnikov and I. Losev, Etingof conjecture for quantized quiver varieties, arXiv: 1309.1716

[BL94] Joseph Bernstein and Valery Lunts, Equivariant sheaves and functors, Lecture Notes in Mathematics, vol. 1578, Springer-Verlag, Berlin, 1994. MR MR1299527 (95k:55012)

[BLPW] Tom Braden, Anthony Licata, Nicholas Proudfoot, and Ben Webster, Quantizations of conical symplectic resolutions II: category $O$, arXiv: 1407.0964.

[BPW] Tom Braden, Nicholas J. Proudfoot, and Ben Webster, Quantizations of conical symplectic resolutions I: local and global structure, arXiv: 1208.3863 .

[Bru] Jon Brundan, On the definition of Kac-Moody 2-category, arXiv: 1501.00350

[CBEG07] William Crawley-Boevey, Pavel Etingof, and Victor Ginzburg, Noncommutative geometry and quiver algebras, Adv. Math. 209 (2007), no. 1, 274-336.

[CDK] Sabin Cautis, Christopher Dodd, and Joel Kamnitzer, in preparation.

[CK] Sabin Cautis and Joel Kamnitzer, Braiding via geometric lie algebra actions, arXiv: $1001.0619 \mathrm{v} 2$

[CKLa] Sabin Cautis, Joel Kamnitzer, and Anthony Licata, Coherent sheaves and categorical sl(2) actions, arXiv:0902.1797.

[CKLb] Coherent sheaves on quiver varieties and categorification, arXiv: 1104.0352

[CKLc] Derived equivalences for cotangent bundles of grassmannians via categorical sI(2) actions, arXiv:0902.1796

[CKL10] Sabin Cautis, Joel Kamnitzer, and Anthony Licata, Categorical geometric skew Howe duality, Invent. Math. 180 (2010), no. 1, 111-159. MR 2593278 (2011d:17021)

[CL15] Sabin Cautis and Aaron D. Lauda, Implicit structure in 2-representations of quantum groups, Selecta Math. (N.S.) 21 (2015), no. 1, 201-244.

[CR08] Joseph Chuang and Raphaël Rouquier, Derived equivalences for symmetric groups and $\mathfrak{s l}_{2}-$ categorification, Ann. of Math. (2) 167 (2008), no. 1, 245-298.

[EGGO07] Pavel Etingof, Wee Liang Gan, Victor Ginzburg, and Alexei Oblomkov, Harish-Chandra homomorphisms and symplectic reflection algebras for wreath-products, Publ. Math. Inst. Hautes Études Sci. (2007), no. 105, 91-155.

[EK10] Ben Elias and Mikhail Khovanov, Diagrammatics for Soergel categories, Int. J. Math. Math. Sci. (2010), Art. ID 978635, 58. MR 3095655

[Eti12] Pavel Etingof, Symplectic reflection algebras and affine Lie algebras, Mosc. Math. J. 12 (2012), no. 3, 543-565, 668-669. 
[EW] Ben Elias and Geordie Willamson, Soergel Calculus, arXiv:1309.0865

[Gor06] Iain Gordon, A remark on rational Cherednik algebras and differential operators on the cyclic quiver, Glasg. Math. J. 48 (2006), no. 1, 145-160.

[KL10] Mikhail Khovanov and Aaron D. Lauda, A categorification of quantum sl(n), Quantum Topol. 1 (2010), no. 1, 1-92. MR 2628852 (2011g:17028)

[KR08] Masaki Kashiwara and Raphaël Rouquier, Microlocalization of rational Cherednik algebras, Duke Math. J. 144 (2008), no. 3, 525-573. MR MR2444305

[KS] Masaki Kashiwara and Pierre Schapira, Deformation quantization modules, arXiv: 1003.3304.

[KW07] Anton Kapustin and Edward Witten, Electric-magnetic duality and the geometric Langlands program, Commun. Number Theory Phys. 1 (2007), no. 1, 1-236.

[Lia] Yiqiang Li, On geometric realizations of quantum modified algebras and their canonical bases, arXiv: 1007.5384

[Lib] _ On geometric realizations of quantum modified algebras and their canonical bases II, arXiv: 1009.0838

[Los12] Ivan Losev, Isomorphisms of quantizations via quantization of resolutions, Adv. in Math. (2012), no. 231, 1216-1270.

[MN] Kevin McGerty and Thomas Nevins, Morse decomposition for D-module categories on stacks, arXiv: 1402.7365 ,

[Nak94] Hiraku Nakajima, Instantons on ALE spaces, quiver varieties, and Kac-Moody algebras, Duke Math. J. 76 (1994), no. 2, 365-416. MR MR1302318 (95i:53051)

[Nak98] _ Quiver varieties and Kac-Moody algebras, Duke Math. J. 91 (1998), no. 3, 515-560. MR MR1604167 (99b:17033)

[NZ09] David Nadler and Eric Zaslow, Constructible sheaves and the Fukaya category, J. Amer. Math. Soc. 22 (2009), no. 1, 233-286.

[Roua] Raphael Rouquier, 2-Kac-Moody algebras, arXiv:0812.5023.

[Roub] Quiver Hecke algebras and 2-Lie algebras, arXiv:1112.3619.

[VV11] Michela Varagnolo and Eric Vasserot, Canonical bases and KLR-algebras, J. Reine Angew. Math. 659 (2011), 67-100.

[Weba] Ben Webster, Centers of KLR algebras and cohomology rings of quiver varieties, arXiv: 1504.04401.

[Webb] Comparison of canonical bases for Schur and universal enveloping algebras., arXiv: 1503.08734

[Webc] Erratum to "Canonical bases and higher representation theory", people.virginia.edu/ btw4e/CB-erratum.pdf

[Webd] , Knot invariants and higher representation theory, arXiv:1309.3796

[Webe] On generalized category $O$ for a quiver variety, arXiv: 1409.4461

[Webf] Weighted Khovanov-Lauda-Rouquier algebras, arXiv:1209.2463

[Web15] Canonical bases and higher representation theory, Compos. Math. 151 (2015), no. 1, 121-166.

[WW] Ben Webster and Geordie Williamson, The bounded below equivariant derived category, In preparation. Preliminary version available at http://people.virginia.edu/ b btw4e/publications.html

[WW10] Katrin Wehrheim and Chris T. Woodward, Functoriality for Lagrangian correspondences in Floer theory, Quantum Topol. 1 (2010), no. 2, 129-170. MR 2657646 (2011g:53193)

[Zhe] Hao Zheng, Categorification of integrable representations of quantum groups, arXiv: 0803.3668. 